\title{
Oxygen trends in the Galactic thin and thick disks ${ }^{\star \star \star}$
}

\author{
T. Bensby, S. Feltzing, and I. Lundström
}

\author{
Lund Observatory, Box 43, 22100 Lund, Sweden
}

Received 21 July 2003 / Accepted 24 October 2003

\begin{abstract}
We present oxygen abundances for $72 \mathrm{~F}$ and $\mathrm{G}$ dwarf stars in the solar neighbourhood. Using the kinematics of the stars we divide them into two sub-samples with space velocities that are typical for the thick and thin disks, respectively. The metallicities of the stars range from $[\mathrm{Fe} / \mathrm{H}] \approx-0.9$ to +0.4 and we use the derived oxygen abundances of the stars to: (1) perform a differential study of the oxygen trends in the thin and the thick disk; (2) to follow the trend of oxygen in the thin disk to the highest metallicities. We analyze the forbidden oxygen lines at $6300 \AA$ and $6363 \AA$ as well as the (NLTE afflicted) triplet lines around $7774 \AA$. For the forbidden line at $6300 \AA$ we have spectra of very high $S / N(>400)$ and resolution $(R \gtrsim 215000)$. This has enabled a very accurate modeling of the oxygen line and the blending Ni lines. The high internal accuracy in our determination of the oxygen abundances from this line is reflected in the very tight trends we find for oxygen relative to iron. From these abundances we are able to draw the following major conclusions: (i) That the $[\mathrm{O} / \mathrm{Fe}]$ trend at super-solar $[\mathrm{Fe} / \mathrm{H}]$ continues downward which is in concordance with models of Galactic chemical evolution. This is not seen in previous studies as it has not been possible to take the blending Ni lines in the forbidden oxygen line at $6300 \AA$ A properly into account; (ii) That the oxygen trends in the thin and the thick disks are distinctly different. This confirms and extends previous studies of the other $\alpha$-elements; (iii) That oxygen does not follow Mg at super-solar metallicities; (iv) We also provide an empirical NLTE correction for the infrared O I triplet that could be used for dwarf star spectra with a $S / N$ such that only the triplet lines can be analyzed well, e.g. stars at large distances; (v) Finally, we find that Gratton et al. (1999) overestimate the NLTE corrections for the permitted oxygen triplet lines at $\sim 7774 \AA$ for the parameter space that our stars span.
\end{abstract}

Key words. stars: fundamental parameters - stars: abundances - galaxy: disk - galaxy: formation - galaxy: abundances galaxy: kinematics and dynamics

\section{Introduction}

Oxygen is, next to hydrogen and helium, the most abundant element in the Universe. Of the three stable isotopes ${ }^{16} \mathrm{O},{ }^{17} \mathrm{O}$, and ${ }^{18} \mathrm{O}$, the first is the dominating one, making up $\sim 99.8 \%$ of the total oxygen content in the Solar system. Oxygen is a bona fide primary element that essentially only forms in the interiors of massive stars through hydrostatic burning of mainly $\mathrm{He}, \mathrm{C}$, and Ne. By analyzing elemental abundances in the atmospheres of long-lived $\mathrm{F}$ and $\mathrm{G}$ dwarf stars it is not only possible to determine the chemical composition of the gas that the stars were born out of but also to trace the chemical history in the different stellar populations in the Milky Way. In this respect the oxygen fossil record is of extra importance and is often used in models of Galactic evolution. It can, among other things, be used to measure the rates of supernovae type II (SN II) and supernovae type Ia (SN Ia) with time (e.g. Wheeler et al. 1989).

Send offprint requests to: T. Bensby, e-mail: thomas@astro.lu. se

* Based on observations collected at the European Southern Observatory, La Silla and Paranal, Chile, Proposals \#65.L-0019, 67.B-0108, and 69.B-0277.

$\star \star$ The full Table 4 is only available in electronic form at the CDS via anonymous ftp to cdsarc.u-strasbg.fr $(130.79 .128 .5)$ or via http://cdsweb.u-strasbg.fr/cgi-bin/qcat?/A+A/415/155
An overabundance of oxygen $\left([\mathrm{O} / \mathrm{Fe}]^{1}>0\right)$ indicates that the region have had a high star formation rate and undergone a fast chemical enrichment (e.g. Tinsley 1979; Matteucci \& Greggio 1986).

The determination of oxygen abundances is unfortunately often troublesome due to the limited number of available oxygen lines in the visual part of a stellar spectrum. The main indicators are the O I triplet at $\sim 7774 \AA$, the forbidden $[\mathrm{OI}]^{2}$ lines, especially those at $6300 \AA$ and $6363 \AA$, the ultraviolet (UV), and the infrared (IR) $\mathrm{OH}$ lines. The analysis of these lines all have their difficulties. The triplet at $7774 \AA$ should be ideal to work with since its lines are strong and are located in a clean part of the spectrum that is free from blending lines. The lines are, however, strongly affected by deviations from local thermal equilibrium (LTE) (see e.g. Kiselman 1991) and, even if these deviations are considered not very well understood even for the Sun, they are probably due to convective inhomogeneities (Kiselman 1993). The forbidden lines are very robust indicators but are hard to work

\footnotetext{
1 Abundances expressed within brackets are as usual relative to solar values: $[\mathrm{O} / \mathrm{Fe}]=\log \left(N_{\mathrm{O}} / N_{\mathrm{Fe}}\right)_{\star}-\log \left(N_{\mathrm{O}} / N_{\mathrm{Fe}}\right)_{\odot}$.

2 These brackets indicate that the spectral line is forbidden and should not be confused with the notation of abundances given relative to solar values.
} 
with since they are both weak (the measured equivalent widths in the Sun are $W_{\lambda}(6300) \approx 5 \mathrm{~m} \AA$ and $W_{\lambda}(6363) \approx 3 \mathrm{~m} \AA$, Moore et al. 1966). Both lines are also blended by lines from other species. The [O I] line at $6300 \AA$ has two Ni I lines in its right wing (Lambert 1978; Johansson et al. 2003) and the [O I] line at $6363 \AA$ has a possible CN contribution (Lambert 1978).

The conclusion from the Joint Discussion 8 during the IAU General Assembly in Manchester in 2000 (New Astronomy Reviews 45, 2001), which was devoted to a discussion of oxygen abundances in old stars, is that the most reliable indicators of stellar oxygen abundances are the forbidden [O I] lines (Barbuy et al. 2001). The [O I] line at $6300 \AA$ is blended and is located in a part of the spectrum that is severely affected by telluric lines. If we want to use this line for abundance analysis it is important to be able to take these effects into account properly. This is best done by using spectra with high signalto-noise ratios $(S / N)$ and high resolutions $(R)$ together with accurate atomic line data, $\log g f$-values in particular.

In the Milky Way it is mainly the halo stellar population that shows the large overabundances of oxygen relative to iron, indicative of a fast star formation. There is however an ongoing controversy about the size of this overabundance. Depending on which indicators that are used in the determination of the oxygen abundances one can find different $[\mathrm{O} / \mathrm{Fe}]$ trends for these stars. The forbidden oxygen line at $6300 \AA$ and the IR $\mathrm{OH}$ lines generally produce a constant value of $[\mathrm{O} / \mathrm{Fe}] \approx 0.5$ for halo stars with $[\mathrm{Fe} / \mathrm{H}] \lesssim-1$, see e.g. Nissen et al. (2002), Gratton \& Ortolani (1986), Sneden et al. (1979), and Lambert et al. (1974) for abundances from the [O I] line at $6300 \AA$, and e.g. Meléndez \& Barbuy (2002), Meléndez et al. (2001), and Balachandran et al. (2001) for abundances from the IR $\mathrm{OH}$ lines. Other indicators such as the O I triplet at $7774 \AA$ and the UV OH lines give higher [O/Fe] values with an uprising trend when going to lower $[\mathrm{Fe} / \mathrm{H}]$, see e.g. Israelian et al. (2001) and Abia \& Rebolo (1989) for abundances from the O I triplet, and Boesgaard et al. (1999) and Israelian et al. (1998) for abundances from the UV OH lines. However, agreement between abundances from the UV OH lines and the forbidden lines has also been found (e.g. Bessel et al. 1991) and also between the $\mathrm{O}$ I triplet and the forbidden lines (e.g. Carretta et al. 2000; Mishenina et al. 2000).

For stars more metal-rich than the halo (i.e. $[\mathrm{Fe} / \mathrm{H}] \gtrsim-1$ to -0.5$)$ the observed trends are further complicated by the fact that there is a mixture of stars from different stellar populations, each with their own chemical history (see e.g. Gratton et al. 2003). The observed $[\mathrm{O} / \mathrm{Fe}]$ trend for the halo stars generally extends to $[\mathrm{Fe} / \mathrm{H}] \sim-0.5$ with an overabundance of $[\mathrm{O} / \mathrm{Fe}] \sim 0.4-0.5$. Nissen \& Schuster (1997), however, found that the most metal-rich halo stars to be less enhanced in $[\mathrm{O} / \mathrm{Fe}]$ than the thick disk stars at the same metallicities. Quite good agreement between the different oxygen indicators are generally found at these metallicities (see works cited above). For the thick disk no (previous) study has included thick disk stars with $[\mathrm{Fe} / \mathrm{H}] \gtrsim-0.35$ when studying the $[\mathrm{O} / \mathrm{Fe}]$ vs. $[\mathrm{Fe} / \mathrm{H}]$ trend. Below this metallicity, thick disk stars are generally found to show an $[\mathrm{O} / \mathrm{Fe}]$ trend similar to the halo trend, i.e. a constant value of $[\mathrm{O} / \mathrm{Fe}] \sim 0.4-$ 0.5 (see e.g. Nissen et al. 2002; Tautvaišienè et al. 2001;
Prochaska et al. 2000; Gratton et al. 2000; Chen et al. 2000). The thin disk stars present small to moderate overabundances of oxygen $(0 \lesssim[\mathrm{O} / \mathrm{Fe}] \lesssim 0.2)$ for metallicities down to $[\mathrm{Fe} / \mathrm{H}] \approx-0.8$ with the largest values of $[\mathrm{O} / \mathrm{Fe}]$ at the lower $[\mathrm{Fe} / \mathrm{H}]$ values (see e.g. Reddy et al. 2003; Nissen et al. 2002; Edvardsson et al. 1993; Nissen \& Edvardsson 1992; Kjærgaard et al. 1982). The distinction between the thin and thick disk is however somewhat unclear. While e.g. Prochaska et al. (2000) found them to be distinct in terms of abundances, Chen et al. (2000) found the elemental abundance trends in the thin and thick disks to follow smoothly upon each other.

At super-solar metallicities $([\mathrm{Fe} / \mathrm{H}]>0)$ a constant value of $[\mathrm{O} / \mathrm{Fe}] \sim 0$ was found by Nissen \& Edvardsson (1992), and Nissen et al. (2002). Feltzing \& Gustafsson (1998) has a somewhat larger scatter in the $[\mathrm{O} / \mathrm{Fe}]$ trend and also find a potential decline towards higher $[\mathrm{Fe} / \mathrm{H}]$. A decline in $[\mathrm{O} / \mathrm{Fe}]$ is supported by the studies of Castro et al. (1997) and Chen et al. (2003), but is at variance with other studies discussed above.

In the solar neighbourhood it is also possible to come across stars which might originate in the Galactic bulge or inner disk (Pompéia et al. 2002). They are generally old (>10 Gyr) and Pompéia et al. (2002) found that they show an overabundance of oxygen when compared to their disk counterparts for metallicities ranging $-0.8 \leq[\mathrm{Fe} / \mathrm{H}] \leq+0.4$.

In this paper we will determine the oxygen abundances for thin and thick disk stars with $-0.9 \lesssim[\mathrm{Fe} / \mathrm{H}] \lesssim+0.4$. The stars have been selected on purely kinematical grounds (see Bensby et al. 2003 for a full discussion of the selection process) in order to address two important questions: 1 ) the $[\mathrm{O} / \mathrm{Fe}]$ trends at $[\mathrm{Fe} / \mathrm{H}] \lesssim 0$ in the thin and thick disks; 2) the $[\mathrm{O} / \mathrm{Fe}]$ trend at super-solar metallicities.

Our oxygen abundances are based on the forbidden [O I] line at $6300 \AA$. In order to properly account for the $\mathrm{Ni}$ blends and the fact that the line generally is very weak we have obtained spectra of very high quality $(R \gtrsim 215000$ and $S / N \gtrsim 400)$. We have used the new $\log g f$ values for the Ni blends from Johansson et al. (2003) who also showed that the Ni blend actually consists of two isotopic Ni components.

The paper is organized as follows. In Sect. 2 we describe the observations and the data reductions. In Sect. 3 we describe the atomic data used in the abundance determination, which in turn is described in Sect. 4. Section 5 deals with the errors in the resulting abundances. The abundance trends that we derive from the different indicators are presented in Sect. 6. In Sect. 7 we put our results into the contexts of Galactic chemical evolution. We finally give a summary in Sect. 8 .

\section{Observations and data reductions}

\subsection{Stellar sample}

The stellar samples have been kinematically selected to contain stars with high probabilities of belonging to either the thin or the thick Galactic disks. These probabilities were derived under the assumption that the stellar space velocities $U_{\mathrm{LSR}}$, $V_{\mathrm{LSR}}$, and $W_{\mathrm{LSR}}$ in the thin and thick disks have Gaussian distributions. For each star it is then possible to calculate the 
Table 1. The 72 program stars. $T_{\text {eff }}, \log g, \xi_{\mathrm{t}},[\mathrm{Fe} / \mathrm{H}]$, and $[\mathrm{Ni} / \mathrm{H}]$ are taken from Bensby et al. (2003). All abundances have been normalized to the Sun. Both NLTE corrected, according to the prescription in Gratton et al. (2000), and un-corrected abundances are given for the triplet lines. For oxygen abundances from the individual triplet lines see Table 4. The last three columns give the spectrographs and exposure times used: $\mathrm{C}=\mathrm{CES}(\mathrm{ESO} 3.6 \mathrm{~m}), \mathrm{F}=$ FEROS (ESO $1.5 \mathrm{~m})$, and U = UVES (ESO VLT/UT2).

\begin{tabular}{|c|c|c|c|c|c|c|c|c|c|c|c|c|c|c|}
\hline \multicolumn{3}{|c|}{ Identifications } & \multirow{2}{*}{$\begin{array}{l}T_{\text {eff }} \\
{[\mathrm{K}]}\end{array}$} & \multirow{2}{*}{$\begin{array}{l}\log g \\
{[\operatorname{cgs}]}\end{array}$} & \multirow{2}{*}{$\begin{array}{c}\xi_{\mathrm{t}} \\
{\left[\mathrm{km} \mathrm{s}^{-1}\right]}\end{array}$} & \multirow{2}{*}{$\begin{array}{l}v_{\text {rot-macro }} \\
{\left[\mathrm{km} \mathrm{s}^{-1}\right]}\end{array}$} & \multirow[t]{2}{*}[\mathrm{Fe}/\mathrm{H}]{} & \multirow[t]{2}{*}[\mathrm{Ni}/\mathrm{H}]{} & \multirow{2}{*}{$\begin{array}{r}{[\mathrm{O} / \mathrm{H}]} \\
6300\end{array}$} & \multirow{2}{*}{$\begin{array}{r}{[\mathrm{O} / \mathrm{H}]} \\
6363\end{array}$} & \multirow{2}{*}{$\begin{array}{r}{[\mathrm{O} / \mathrm{H}]} \\
7774\end{array}$} & \multirow{2}{*}{\multicolumn{3}{|c|}{$\begin{array}{c}\text { Spectrograph (EXPTIME) } \\
{[\text { min }]}\end{array}$}} \\
\hline Hip & HD & HR & & & & & & & & & & & & \\
\hline \multicolumn{15}{|c|}{ THIN DISK STARS } \\
\hline Sun & & & 5777 & 4.44 & 0.85 & 1.74 & 0.00 & 0.00 & 0.00 & 0.00 & 0.00 & $\mathrm{C}$ & $\mathrm{F}$ & $\mathrm{U}$ \\
\hline 3142 & 3735 & 170 & 6100 & 4.07 & 1.50 & 2.22 & -0.45 & -0.47 & -0.25 & & -0.19 & $\mathrm{C}(80)$ & $\mathrm{F}(9)$ & \\
\hline 3170 & 3823 & 176 & 5970 & 4.11 & 1.40 & 2.13 & -0.34 & -0.37 & & & -0.06 & C (30) & $\mathrm{F}(10)$ & \\
\hline 5862 & 7570 & 370 & 6100 & 4.26 & 1.10 & 2.80 & 0.17 & 0.16 & 0.06 & & & $\mathrm{C}(20)$ & & $\mathrm{U}(0.3)$ \\
\hline 7276 & 9562 & 448 & 5930 & 3.99 & 1.35 & 2.61 & 0.20 & 0.24 & 0.14 & & 0.12 & $\mathrm{C}(30)$ & $\mathrm{F}(4)$ & \\
\hline 9085 & 12042 & 573 & 6200 & 4.25 & 1.30 & 3.19 & -0.31 & -0.37 & & & -0.05 & C (28) & $F(6.3)$ & $\mathrm{U}(1)$ \\
\hline 10798 & 14412 & 683 & 5350 & 4.57 & 0.20 & 1.51 & -0.47 & -0.48 & -0.30 & & -0.37 & $\mathrm{C}(45)$ & $F(7)$ & \\
\hline 12186 & 16417 & 772 & 5800 & 4.04 & 1.20 & 1.94 & 0.14 & 0.14 & 0.08 & & 0.13 & C (30) & $\mathrm{F}(4.3)$ & \\
\hline 12611 & 17006 & 807 & 5250 & 3.66 & 1.35 & 2.51 & 0.26 & 0.33 & & 0.14 & 0.34 & C (35) & $F(6)$ & \\
\hline 12653 & 17051 & 810 & 6150 & 4.37 & 1.25 & 3.43 & 0.14 & 0.12 & 0.04 & 0.06 & 0.20 & $C(16)$ & F (3) & \\
\hline 14954 & 19994 & 962 & 6240 & 4.10 & 1.60 & 4.40 & 0.19 & 0.22 & 0.11 & & 0.18 & C (40) & F (3) & \\
\hline 15131 & 20407 & & 5834 & 4.35 & 1.00 & 1.58 & -0.52 & -0.52 & -0.26 & & & & & $\mathrm{U}(1.5)$ \\
\hline 17378 & 23249 & 1136 & 5020 & 3.73 & 0.80 & 1.80 & 0.24 & 0.31 & 0.13 & 0.05 & 0.08 & $C(12)$ & $F(0.5)$ & \\
\hline 22263 & 30495 & 1532 & 5850 & 4.50 & 0.95 & 2.40 & 0.05 & -0.01 & & & 0.06 & $\mathrm{C}(21)$ & $\mathrm{F}(3.3)$ & \\
\hline 22325 & 30606 & 1538 & 6250 & 3.91 & 1.80 & 3.90 & 0.06 & 0.02 & 0.01 & & 0.20 & C (108) & F (4) & \\
\hline 23555 & 32820 & 1651 & 6300 & 4.29 & 1.50 & 4.58 & 0.13 & 0.11 & 0.06 & & 0.21 & C (34) & $F(6.7)$ & \\
\hline 23941 & 33256 & 1673 & 6427 & 4.04 & 1.90 & 5.20 & -0.30 & -0.36 & & & -0.06 & & $F(4.7)$ & \\
\hline 24829 & 35072 & 1767 & 6360 & 3.93 & 1.70 & 2.37 & 0.06 & 0.01 & 0.03 & & 0.16 & $C(20)$ & $\mathrm{F}(3.3)$ & \\
\hline 29271 & 43834 & 2261 & 5550 & 4.38 & 0.80 & 1.68 & 0.10 & 0.16 & 0.02 & & 0.15 & $C(20)$ & $\mathrm{F}(3)$ & \\
\hline 30480 & 45701 & 2354 & 5890 & 4.15 & 1.20 & 2.11 & 0.19 & 0.22 & 0.17 & 0.10 & 0.20 & $C(50)$ & $F(8.3)$ & \\
\hline 30503 & 45184 & 2318 & 5820 & 4.37 & 0.90 & 2.24 & 0.04 & 0.02 & 0.02 & 0.00 & 0.10 & C (41) & $F(8.3)$ & \\
\hline 72673 & 130551 & & 6350 & 4.18 & 1.60 & 2.30 & -0.62 & -0.60 & -0.30 & & -0.34 & C (80) & $F(15)$ & \\
\hline 78955 & 144585 & 5996 & 5880 & 4.22 & 1.12 & 2.32 & 0.33 & 0.40 & 0.17 & 0.17 & 0.23 & $C(60)$ & $F(6)$ & \\
\hline 80337 & 147513 & 6094 & 5880 & 4.49 & 1.10 & 2.09 & 0.03 & -0.04 & 0.03 & & -0.04 & $\mathrm{C}(20)$ & F (3) & \\
\hline 80686 & 147584 & 6098 & 6090 & 4.45 & 1.01 & 2.28 & -0.06 & -0.13 & -0.05 & -0.09 & 0.01 & $C(20)$ & $F(2)$ & \\
\hline 81520 & 149612 & & 5680 & 4.53 & 0.65 & 1.70 & -0.48 & -0.49 & & & -0.24 & & $\mathrm{~F}(11)$ & \\
\hline 83601 & 154417 & 6349 & 6167 & 4.48 & 1.21 & 3.27 & 0.09 & 0.00 & 0.07 & & 0.04 & C (34) & $\mathrm{F}(5)$ & \\
\hline 84551 & 156098 & 6409 & 6475 & 3.79 & 2.00 & 3.98 & 0.12 & 0.08 & 0.12 & & 0.28 & C (18) & $F(3.3)$ & \\
\hline 84636 & 156365 & & 5820 & 3.91 & 1.30 & 2.68 & 0.23 & 0.32 & 0.15 & & 0.28 & C (55) & $F(9)$ & \\
\hline 85007 & 157466 & & 6050 & 4.37 & 1.10 & 2.43 & -0.39 & -0.45 & -0.26 & & -0.27 & C (75) & $F(10)$ & \\
\hline 85042 & 157347 & 6465 & 5720 & 4.40 & 1.00 & 1.65 & 0.03 & 0.06 & 0.00 & 0.06 & -0.05 & C (40) & $F(6.7)$ & \\
\hline 86731 & 161239 & 6608 & 5840 & 3.79 & 1.43 & 3.37 & 0.25 & 0.33 & & & 0.26 & C (39) & $F(5)$ & \\
\hline 86796 & 160691 & 6585 & 5800 & 4.30 & 1.05 & 2.20 & 0.32 & 0.41 & 0.14 & & 0.24 & C (27) & $F(2.5)$ & \\
\hline 87523 & 162396 & 6649 & 6070 & 4.07 & 1.36 & 2.17 & -0.40 & -0.39 & -0.31 & -0.31 & -0.23 & C (35) & $F(6)$ & \\
\hline 90485 & 169830 & 6907 & 6339 & 4.05 & 1.55 & 2.59 & 0.12 & 0.11 & & 0.06 & 0.25 & C (31) & F (5) & \\
\hline 91438 & 172051 & 6998 & 5580 & 4.42 & 0.55 & 1.62 & -0.24 & -0.26 & -0.18 & & -0.23 & C (45) & F (4) & \\
\hline 94645 & 179949 & 7291 & 6200 & 4.35 & 1.20 & 3.90 & 0.16 & 0.11 & 0.06 & 0.11 & 0.23 & C (40) & $F(6.5)$ & \\
\hline 95447 & 182572 & 7373 & 5600 & 4.13 & 1.10 & 2.14 & 0.37 & 0.47 & 0.20 & 0.23 & 0.38 & C (18) & $F(2.5)$ & \\
\hline 96536 & 184985 & 7454 & 6397 & 4.06 & 1.65 & 2.72 & 0.03 & 0.00 & 0.05 & & 0.20 & $\mathrm{C}(21)$ & $\mathrm{F}(3.3)$ & \\
\hline 98785 & 190009 & 7658 & 6430 & 3.97 & 1.90 & 4.25 & 0.03 & -0.03 & 0.11 & 0.01 & 0.18 & C (60) & $F(7.5)$ & \\
\hline 99240 & 190248 & 7665 & 5585 & 4.26 & 0.98 & 1.72 & 0.37 & 0.44 & 0.19 & 0.17 & 0.32 & C (4) & $\mathrm{F}(1)$ & \\
\hline 100412 & 193307 & 7766 & 5960 & 4.06 & 1.20 & 2.18 & -0.32 & -0.36 & -0.18 & & -0.03 & C (45) & $F(6)$ & \\
\hline 102264 & 197214 & & 5570 & 4.37 & 0.60 & 1.81 & -0.22 & -0.26 & -0.12 & & -0.15 & C (80) & $\mathrm{F}(11)$ & \\
\hline 103682 & 199960 & 8041 & 5940 & 4.26 & 1.25 & 2.34 & 0.27 & 0.35 & 0.14 & 0.12 & 0.21 & C (70) & $F(6.5)$ & \\
\hline 105858 & 203608 & 8181 & 6067 & 4.27 & 1.17 & 2.04 & -0.73 & -0.74 & -0.46 & & -0.34 & $\mathrm{C}(30)$ & $\mathrm{F}(1)$ & \\
\hline 109378 & 210277 & & 5500 & 4.30 & 0.78 & 1.71 & 0.22 & 0.26 & 0.16 & 0.16 & 0.24 & $C(60)$ & $F(8)$ & \\
\hline 110341 & 211976 & 8514 & 6500 & 4.29 & 1.70 & 3.63 & -0.17 & -0.24 & -0.11 & -0.12 & -0.01 & $\mathrm{C}(45)$ & $\mathrm{F}(6)$ & \\
\hline 113137 & 216437 & 8701 & 5800 & 4.10 & 1.16 & 2.17 & 0.22 & 0.27 & 0.13 & 0.12 & 0.27 & C (35) & $F(5.5)$ & \\
\hline 113357 & 217014 & 8729 & 5789 & 4.34 & 1.00 & 1.91 & 0.20 & 0.26 & 0.10 & 0.24 & 0.11 & $\mathrm{C}(21)$ & $F(3.2)$ & \\
\hline 113421 & 217107 & 8734 & 5620 & 4.29 & 0.97 & 1.75 & 0.35 & 0.42 & 0.16 & & 0.27 & $\mathrm{C}(45)$ & $F(6)$ & \\
\hline 117880 & 224022 & 9046 & 6100 & 4.21 & 1.30 & 3.16 & 0.12 & 0.14 & 0.05 & & 0.16 & $C(60)$ & $F(5.5)$ & \\
\hline
\end{tabular}

probability that it kinematically belongs to either the thin or the thick disk. Since we are working with nearby stars we also need to take the local number density of the thin and thick disk stars into consideration. For the thick disk we used a density of $6 \%$ in the solar neighbourhood and consequently $94 \%$ for the thin disk. We then formed the $T D / D$ ratio (i.e. the thick disk probability divided by the thin disk probability) for each star. All thin disk stars in the sample (apart from three) have thin disk probabilities that are more than ten times larger than their thick disk probability (i.e. $T D / D<0.1$ ). Three thin disk stars (Hip 3170, 95447, 100412) have higher $T D / D$ ratios (but still approximately two times more probable of belonging to the 
Table 1. continued.

\begin{tabular}{|c|c|c|c|c|c|c|c|c|c|c|c|c|c|}
\hline \multicolumn{3}{|c|}{ Identifications } & \multirow{2}{*}{$\begin{array}{l}T_{\text {eff }} \\
{[\mathrm{K}]}\end{array}$} & \multirow{2}{*}{$\begin{array}{l}\log g \\
\text { [cgs] }\end{array}$} & \multirow{2}{*}{$\begin{array}{c}\xi_{\mathrm{t}} \\
{\left[\mathrm{km} \mathrm{s}^{-1}\right]}\end{array}$} & \multirow{2}{*}{$\begin{array}{l}v_{\text {rot-macro }} \\
{\left[\mathrm{km} \mathrm{s}^{-1}\right]}\end{array}$} & \multirow[t]{2}{*}[\mathrm{Fe}/\mathrm{H}]{} & \multirow[t]{2}{*}[\mathrm{Ni}/\mathrm{H}]{} & \multirow{2}{*}{$\begin{array}{r}{[\mathrm{O} / \mathrm{H}]} \\
6300\end{array}$} & \multirow{2}{*}{$\begin{array}{r}{[\mathrm{O} / \mathrm{H}]} \\
6363\end{array}$} & \multirow{2}{*}{$\begin{array}{r}{[\mathrm{O} / \mathrm{H}]} \\
7774\end{array}$} & \multirow{2}{*}{\multicolumn{2}{|c|}{$\begin{array}{c}\text { Spectrograph (EXPTIME) } \\
{[\mathrm{min}]}\end{array}$}} \\
\hline Hip & HD & $\mathrm{HR}$ & & & & & & & & & & & \\
\hline \multicolumn{14}{|c|}{ THICK DISK STARS } \\
\hline 3086 & 3628 & & 5840 & 4.15 & 1.15 & 1.90 & -0.11 & -0.12 & 0.09 & & -0.04 & $C(100)$ & $\mathrm{F}(10)$ \\
\hline 3185 & 3795 & 173 & 5320 & 3.78 & 0.70 & 1.64 & -0.59 & -0.57 & -0.14 & -0.21 & -0.11 & $\mathrm{C}(45)$ & $F(6.7)$ \\
\hline 3497 & 4308 & & 5636 & 4.30 & 0.80 & 1.76 & -0.33 & -0.31 & -0.05 & -0.10 & 0.03 & $\mathrm{C}(45)$ & $\mathrm{F}(10)$ \\
\hline 3704 & 4597 & & 6040 & 4.30 & 1.08 & 2.10 & -0.38 & -0.40 & -0.08 & & -0.25 & $\mathrm{C}(180)$ & $\mathrm{F}(10)$ \\
\hline 5315 & 6734 & & 5030 & 3.46 & 0.86 & 1.66 & -0.42 & -0.36 & 0.00 & -0.01 & 0.03 & $\mathrm{C}(45)$ & $\mathrm{F}(10)$ \\
\hline 14086 & 18907 & 914 & 5110 & 3.51 & 0.87 & 1.64 & -0.59 & -0.53 & -0.16 & & -0.20 & C (42) & $F(5)$ \\
\hline 15510 & 20794 & 1008 & 5480 & 4.43 & 0.75 & 1.28 & -0.41 & -0.36 & -0.01 & -0.10 & & $\mathrm{C}(50)$ & $\mathrm{U}(0.2)$ \\
\hline 17147 & 22879 & & 5920 & 4.33 & 1.20 & 1.74 & -0.84 & -0.83 & -0.32 & & -0.32 & $\mathrm{C}(107)$ & $\mathrm{F}(10)$ \\
\hline 75181 & 136352 & 5699 & 5650 & 4.30 & 0.78 & 1.69 & -0.34 & -0.34 & 0.00 & & 0.01 & C (22) & $F(5)$ \\
\hline 79137 & 145148 & 6014 & 4900 & 3.62 & 0.60 & 1.53 & 0.30 & 0.39 & 0.20 & 0.13 & 0.36 & $\mathrm{C}(28)$ & $\mathrm{F}(4)$ \\
\hline 82588 & 152391 & & 5470 & 4.55 & 0.90 & 2.53 & -0.02 & -0.06 & 0.01 & 0.00 & -0.01 & C (54) & $\mathrm{F}(8)$ \\
\hline 83229 & 153075 & & 5770 & 4.17 & 0.97 & 1.52 & -0.57 & -0.51 & & -0.23 & -0.10 & & $\mathrm{~F}(11)$ \\
\hline 84905 & 157089 & & 5830 & 4.06 & 1.20 & 1.98 & -0.57 & -0.56 & -0.15 & & -0.17 & C (75) & $\mathrm{F}(11)$ \\
\hline 88622 & 165401 & & 5720 & 4.35 & 0.80 & 2.10 & -0.46 & -0.45 & -0.05 & -0.09 & 0.02 & C (60) & $F(9)$ \\
\hline 96124 & 183877 & & 5590 & 4.37 & 0.78 & 1.78 & -0.20 & -0.17 & 0.08 & 0.08 & 0.07 & C (80) & F (13) \\
\hline 98767 & 190360 & 7670 & 5490 & 4.23 & 0.66 & 1.98 & 0.25 & 0.31 & 0.21 & 0.13 & 0.38 & C (25) & $F(5)$ \\
\hline 103458 & 199288 & & 5780 & 4.30 & 0.90 & 1.69 & -0.65 & -0.64 & -0.29 & & -0.29 & C (60) & $\mathrm{F}(8)$ \\
\hline 108736 & 208988 & & 5890 & 4.24 & 1.05 & 1.97 & -0.38 & -0.35 & -0.01 & -0.01 & 0.06 & C (80) & $\mathrm{F}(14)$ \\
\hline 109450 & 210483 & & 5830 & 4.18 & 1.10 & 1.43 & -0.13 & -0.15 & 0.05 & 0.08 & 0.06 & & $\mathrm{~F}(10)$ \\
\hline 109821 & 210918 & 8477 & 5800 & 4.29 & 1.05 & 1.72 & -0.08 & -0.10 & 0.10 & & 0.01 & C (45) & $\mathrm{F}(6.7)$ \\
\hline 110512 & 212231 & & 5770 & 4.15 & 1.05 & 2.12 & -0.30 & -0.28 & 0.00 & & 0.07 & C (180) & $\mathrm{F}(10)$ \\
\hline 118115 & 224383 & & 5800 & 4.30 & 1.00 & 1.20 & -0.01 & -0.04 & 0.04 & & -0.01 & & $\mathrm{~F}(10)$ \\
\hline
\end{tabular}

thin disk than the thick disk). The majority of the thick disk stars are more than ten times more probable to belong to the thick disk than to the thin disk (i.e. $T D / D>10$ ). For a few thick disk stars we loosened this criterion somewhat in order to increase the statistics, allowing thick disk stars with $1<T D / D<10$ in the sample. Since the two thick disk subsamples $(T D / D>10$ and $1<T D / D<10)$ show exactly the same trends for other elements such as $\mathrm{Na}, \mathrm{Mg}, \mathrm{Al}, \mathrm{Si}, \mathrm{Ca}, \mathrm{Ti}$, $\mathrm{Cr}, \mathrm{Ni}$, and $\mathrm{Zn}$ (see Bensby et al. 2003), we will not distinguish between them in this study.

Apart from the three thin disk stars with slightly larger $T D / D$ ratios, two additional thin disk stars (Hip 5862 and Hip 15131), and one additional thick disk star (Hip 15510) the stellar sample is the same as in Bensby et al. (2003) (i.e., 66 stars in common). The sample now consists of 22 thick disk stars and 50 thin disk stars (see Table 1). The selection criteria and the stellar samples are fully described in Bensby et al. (2003). The additional 6 stars are described in a forthcoming paper (Bensby et al., in prep.) where we moreover analyze a further 30 thin and thick disk dwarf stars in the northern hemisphere, observed with the SOFIN spectrograph on the NOT telescope on La Palma.

\subsection{Observations}

Observations were carried out with ESO's 3.6 m telescope on La Silla in Chile during two observing runs in September 2000 (4 nights, TB and SF as observers) and August/September 2001 (6 nights, TB as observer). We used the Coudé Echelle Spectrograph (CES) at its highest resolution, $R \simeq 215000$, centered on the forbidden oxygen line at $6300 \AA$ (hereafter denoted as $\left.[\mathrm{OI}]_{6300}\right)$. This setting gives a spectrum with a wavelength coverage of $\sim 40 \AA$. All spectra have a $S / N$ of at least 400 . Long exposures times (given in Table 1) were usually split into three exposures. Since telluric lines are present in this wavelength region we also observed rapidly rotating B stars. These spectra were then used to divide out telluric lines from the object spectra.

Spectra covering the whole optical wavelength region from $3560 \AA$ to $9200 \AA$ were also obtained with the FEROS spectrograph on the ESO $1.5 \mathrm{~m}$ telescope during the same observing runs. These spectra have $R \sim 48000$ and $S / N \gtrsim 150$ and provide $\mathrm{Fe}$ and $\mathrm{Ni}$ abundances as well as the atmospheric parameters for the stars (analysis presented in Bensby et al. 2003).

Spectra for the three additional stars (Hip 5862, 15131, 15510) were obtained with the UVES spectrograph on the VLT/UT2 telescope Kueyen on Paranal in Chile in July 2002 (TB as observer). These spectra have $R \sim 110000$ and $S / N \sim 350$ (Bensby et al., in prep.).

\subsection{Reduction of CES spectra}

The reduction procedure from raw images to wavelength calibrated spectra was carried out with the MIDAS ${ }^{3}$ software. The reductions are straightforward using standard routines concerning cosmic ray filtering, background subtraction, flatfield division, wavelength calibration, and continuum fitting. All exposures for a single star were reduced individually and then the extracted spectra were co-added before the setting of the continuum. For those stars that had a telluric absorption line coinciding with the $[\mathrm{OI}]_{6300}$ line we divided the spectra with a featureless spectrum from a fast rotating B star. Finally the co-added spectra were shifted to rest wavelengths by measuring the doppler shift of eight evenly distributed Fe I lines that have accurate wavelengths from Nave et al. (1994). There are, however, some properties of the CES spectra that are worth discussing in further detail.

3 ESO-MIDAS is the acronym for the European Southern Observatory Munich Image Data Analysis System which is developed and maintained by the European Southern Observatory. 


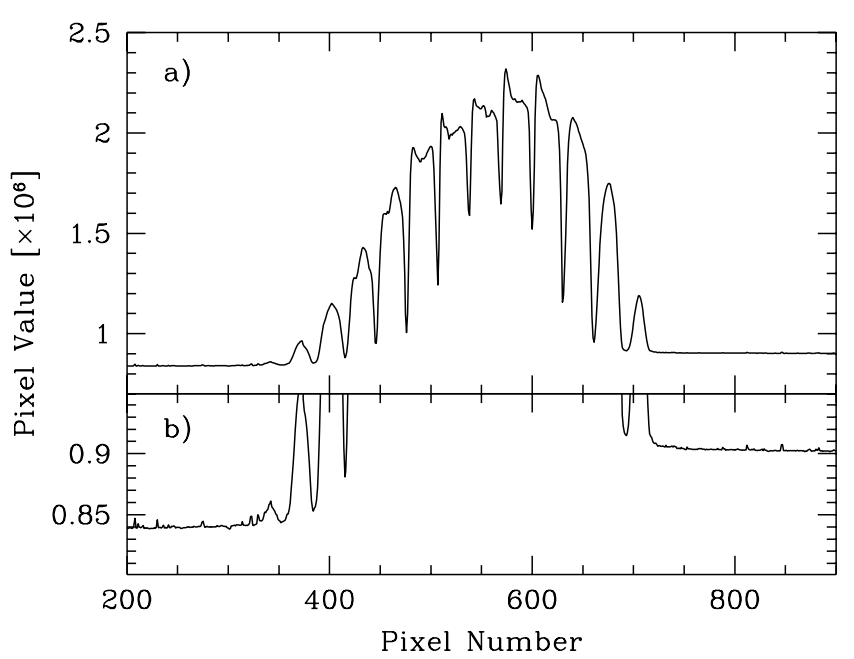

Fig. 1. a) The CES image slicer profile. b) A zoom in on the straylight pedestal beneath the light pattern of the image slicer profile.

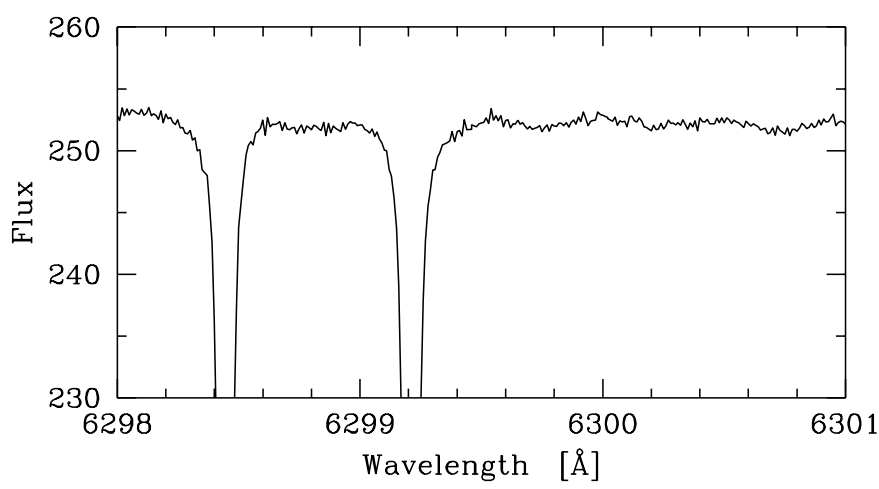

Fig. 2. Example of the interference pattern that originates from reflections within the optical fiber. It is best seen in a featureless spectrum, in this case a fast rotating B star. The two telluric lines are the same as those in Fig. 3. The spectrum has been wavelength calibrated but not normalized and not corrected for the radial velocity of the star.

Straylight pedestal: The background level is different on the two sides of the spectrum in the direction perpendicular to the dispersion direction (see Fig. 1). This is most likely diffuse light produced by the image slicer itself (Kürster 2001). In the wavelength direction this background is slightly nonuniform. Actually it is a low-resolution version of the stellar spectrum that for all practical purposes can be treated as featureless (Kürster 2001). The [O I $]_{6300}$ line profile from individual slices were checked to see if this straylight pedestal had any influence on the line profile and strength from different slices. We especially checked for variations in the line strength (equivalent width) of the $[\mathrm{OI}]_{6300}$ line. No discernible effects (apart from differences in the $S / N$ ) were, however, found between the slices.

Interference pattern: A regular sinusoidal pattern can also be seen in the continuum of a featureless CES spectrum (see Fig. 2). Most likely this has its origin from reflections within the optical fiber that feeds the spectrograph (Kürster 2001). For low metallicity stars with a low density of spectral lines it is possible to perform a Fourier analysis of the pattern and

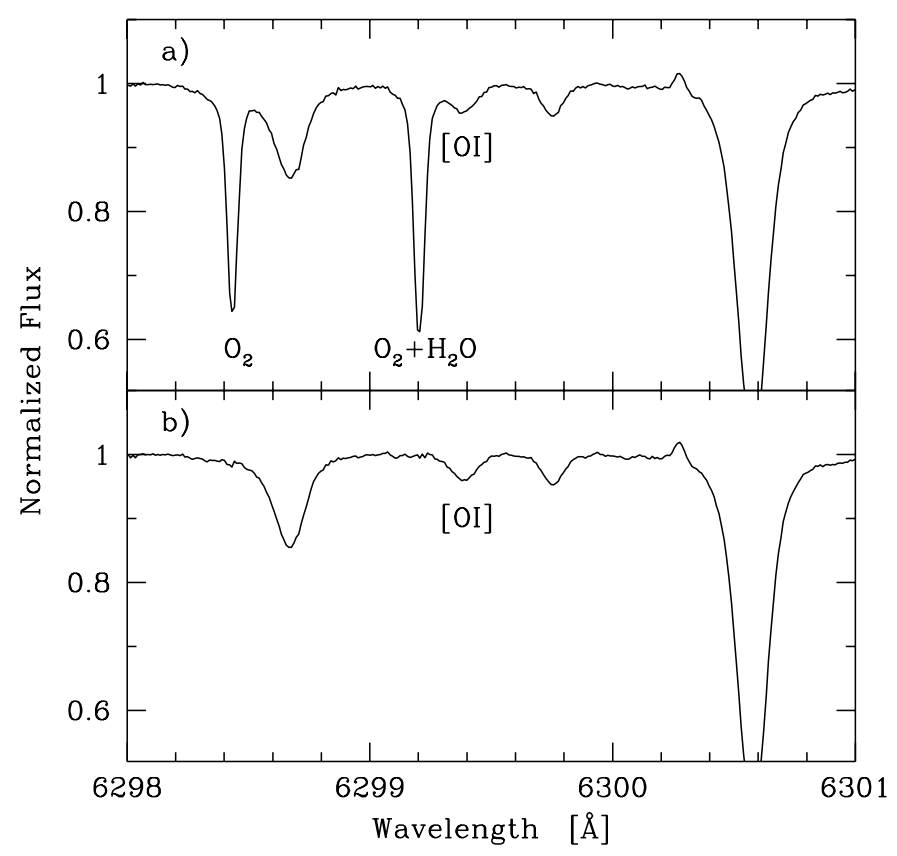

Fig. 3. Example of the removal of lines originating in Earth's atmosphere. The telluric lines, $\mathrm{O}_{2}$ at $6298.5 \AA$, and $\mathrm{O}_{2}+\mathrm{H}_{2} \mathrm{O}$ at $6299.2 \AA$ are indicated as well as the $[\mathrm{OI}]_{6300}$ line. a) Spectrum of Hip 75181 prior to removal of telluric lines and $\mathbf{b}$ ) after removal of telluric lines by division with a spectrum of a fast rotating B star. The spectrum has been wavelength calibrated but not corrected for the radial velocity of the star. Note the telluric oxygen emission line at $\sim 6300.25 \AA$.

remove it. However, most of our stars are rich in spectral lines which makes a removal nearly impossible. Our B star spectra indicate that the amplitude of this pattern is very small, typically $0.2 \%$ of the continuum flux (see Fig. 2). Unless spectra with $S / N>500$ are striven for, it is therefore safe to ignore this feature in the reduction process.

Telluric absorption lines: Telluric lines were divided out using spectra of fast rotating B stars with the IRAF ${ }^{4}$ task TELLURIC which is part of the NOAO package. The procedure takes care of small possible shifts in the dispersion zero-points as well as of the variations in the abundance of the telluric species by shifting and scaling the B star spectrum. An example of the removal of the telluric absorption lines is given in Fig. 3. Of the 72 stars in our sample there were eight stars (Hip 3086, $3185,3704,15510,75181,82588,88622$, and 95447) that had a telluric absorption line that blended the $[\mathrm{OI}]_{6300}$ line. Only for these eight stars did we divide out the telluric lines.

Telluric emission lines: Unfortunately there were also occasions when the telluric oxygen emission line (see Fig. 3) coincides with the stellar $[\mathrm{OI}]_{6300}$ line. Since the presence and strength of this feature vary strongly with time it is very difficult to remove. A possible way to remove it would be to

${ }^{4}$ IRAF is distributed by National Optical Astronomy Observatories, operated by the Association of Universities for Research in Astronomy, Inc., under contract with the National Science Foundation, USA. 
observe a part of the sky next to the object immediately after the observation of the star, and then use this sky spectrum to divide out the sky emission. We did, however, not obtain such sky spectra. For seven stars (Hip 3170, 9085, 12611, 22263, $86731,90485,83229)$ we were unable to determine the oxygen abundance based on the $[\mathrm{OI}]_{6300}$ line due to this telluric emission feature.

\section{Atomic data}

The oscillator strength of the weak Ni line at $6300.35 \AA$ has recently been measured in the laboratory by Johansson et al. (2003). They showed that it actually contains two isotopic components, $\lambda\left({ }^{58} \mathrm{Ni}\right)=6300.335 \AA$ and $\lambda\left({ }^{60} \mathrm{Ni}\right)=6300.355 \AA$. Both components have $\log g f=-2.11$. Assuming a solar isotopic ratio of 0.38 for the abundances of ${ }^{60} \mathrm{Ni}$ and ${ }^{58} \mathrm{Ni}$ and that these two isotopes make up $94.4 \%$ of the total $\mathrm{Ni}$ abundance (CRC 2002), the weighted $\log g f$-values for these isotopes are $\log g f\left({ }^{58} \mathrm{Ni}\right)=-2.275$ and $\log g f\left({ }^{60} \mathrm{Ni}\right)=-2.695$.

The $\log g f$-values for the oxygen lines have been taken from the VALD database (Piskunov et al. 1995; Kupka et al. 1999; Ryabchikova et al. 1999). The source for the [O I] lines is the compilation of Wiese et al. (1966) who based the transition probabilities on theoretical calculations from Stoffregen \& Derblom (1960), Omholt (1960), Naqvi (1951), and Yamanouchi \& Horie (1952). For the O I triplet lines the original sources are Biémont \& Zeippen (1992) and Hibbert et al. (1991).

The broadening of atomic lines by radiation damping was considered in the determination of the abundances and the damping constants $\left(\gamma_{\mathrm{rad}}\right)$ for the different lines were collected from the VALD database.

Collisional broadening, or Van der Waals broadening, was also considered. The width cross-sections are taken from Anstee \& O'Mara (1995), Barklem \& O’Mara (1997, 1998), and Barklem et al. (1998, 2000). Lines present in these works have been marked by an " $S$ " in Table 2 . For spectral lines not present in these works (marked by a "U" in Table 2) we apply the correction term $\left(\delta \gamma_{6}\right)$ to the classical Unsöld approximation of the Van der Waals damping. Following Mäckle et al. (1975) this parameter was set to 2.5 for the lines considered here.

The atomic data are summarized in Table 2.

\section{Abundance determination}

Oxygen abundances have been determined by line synthesis of the $[\mathrm{OI}]_{6300}$ line (Sect. 4.4), by equivalent width measurements of the $[\mathrm{OI}]_{6363}$ line (Sect. 4.5) and the O I triplet lines at $\sim 7774 \AA$ (Sect. 4.6). The actual generation of synthetic spectra was done with the Uppsala SPECTRUM synthesis program and for the calculation of elemental abundances from measured equivalent widths we used the Uppsala EQWIDTH abundance program. The stellar model atmospheres are standard 1D LTE and were calculated with the Uppsala MARCS code (Gustafsson et al. 1975; Edvardsson et al. 1993; Asplund et al. 1997).
Table 2. Atomic line data for the different oxygen lines and the blending Ni lines. Column 1 gives the element, Col. 2 the wavelength, Col. 3 the lower excitation potential, and Col. 4 the correction factor to the classical Unsöld damping constant. Column 5 indicates if the broadening by collisions have been taken from Anstee \& O'Mara (1995), Barklem \& O’Mara (1997, 1998), and Barklem et al. (1998, 2000) (indicated by an "S") instead of the classical Unsöld broadening (indicated by an "U"). Column 6 gives the radiation damping constant and Col. 7 the $\log g f$-values (references are given in Sect. 3).

\begin{tabular}{ccccccc}
\hline \hline El. & $\begin{array}{c}\lambda \\
(\AA)\end{array}$ & $\begin{array}{c}\chi_{1} \\
(\mathrm{eV})\end{array}$ & $\delta \gamma_{6}$ & DMP & $\begin{array}{c}\gamma_{\text {rad }} \\
\left(\mathrm{s}^{-1}\right)\end{array}$ & $\log g f$ \\
\hline$[\mathrm{O}$ I] & 6300.304 & 0.00 & 2.50 & $\mathrm{U}$ & $1.0 \mathrm{e}+05$ & -9.819 \\
{$[\mathrm{O}$ I $]$} & 6363.776 & 0.02 & 2.50 & $\mathrm{U}$ & $1.0 \mathrm{e}+05$ & -10.303 \\
O I & 7771.944 & 9.14 & 2.50 & $\mathrm{~S}$ & $4.8 \mathrm{e}+07$ & 0.37 \\
O I & 7774.166 & 9.14 & 2.50 & $\mathrm{~S}$ & $4.7 \mathrm{e}+07$ & 0.22 \\
O I & 7775.388 & 9.14 & 2.50 & $\mathrm{~S}$ & $4.5 \mathrm{e}+07$ & 0.00 \\
${ }^{58} \mathrm{Ni}$ & 6300.335 & 4.27 & 2.50 & $\mathrm{U}$ & $2.7 \mathrm{e}+08$ & -2.275 \\
${ }^{60} \mathrm{Ni}$ & 6300.355 & 4.27 & 2.50 & $\mathrm{U}$ & $2.7 \mathrm{e}+08$ & -2.695 \\
\hline
\end{tabular}

\subsection{Stellar atmosphere parameters}

Stellar model atmosphere parameters for 66 of the 72 stars in the sample were derived in Bensby et al. (2003) using $\sim 150 \mathrm{Fe}$ I lines measured in the FEROS spectra. The parameters for the additional 6 stars that are included here have been determined in the same way (using UVES spectra for 3 of them) and will be presented in Bensby et al. (in prep.) (together with the SOFIN stars, see Sect. 2). In summary, the tuning of the model atmospheres includes the following steps: effective temperatures were determined by requiring Fe I lines with different lower excitation potentials to produce equal abundances; surface gravities were determined using Hipparcos parallaxes and stellar masses; microturbulences were determined by forcing the $\mathrm{Fe}$ I lines to give the same abundances regardless of line strength. The atmospheric parameters $\left([\mathrm{Fe} / \mathrm{H}], T_{\text {eff }}, \log g\right.$, and $\xi_{\mathrm{t}}$ ) for the whole sample are given together with the derived oxygen abundances in Table 1.

\subsection{Macroscopic line broadening}

Apart from line broadening due to atomic properties the line profile is affected by the instrument profile, the line-of-sight component of the stellar rotation $\left(v_{\mathrm{rot}} \cdot \sin i\right)$, and the macroturbulence. The instrument profile broadening is set by the resolution of the spectra $(R)$. To determine the contributions from the other two broadening mechanisms we used the two strong Fe I lines at $6301.501 \AA$ and $6302.494 \AA$ (the former can be seen to the right in Fig. 3). So, in order to broaden the spectral lines we first convolved the synthetic spectra with a Gaussian profile in order to produce the instrumental broadening and then with an elliptical profile to produce the combined effect of the stellar rotation and macroturbulence. Since we have chosen to include the macroturbulence broadening into the elliptical broadening of the rotation we refer to this profile as $v_{\text {rot-macro }}$.

In Fig. 4 we illustrate the process for Hip 75181. The first step was to tune the $\log g f$-values of the two Fe I lines in 


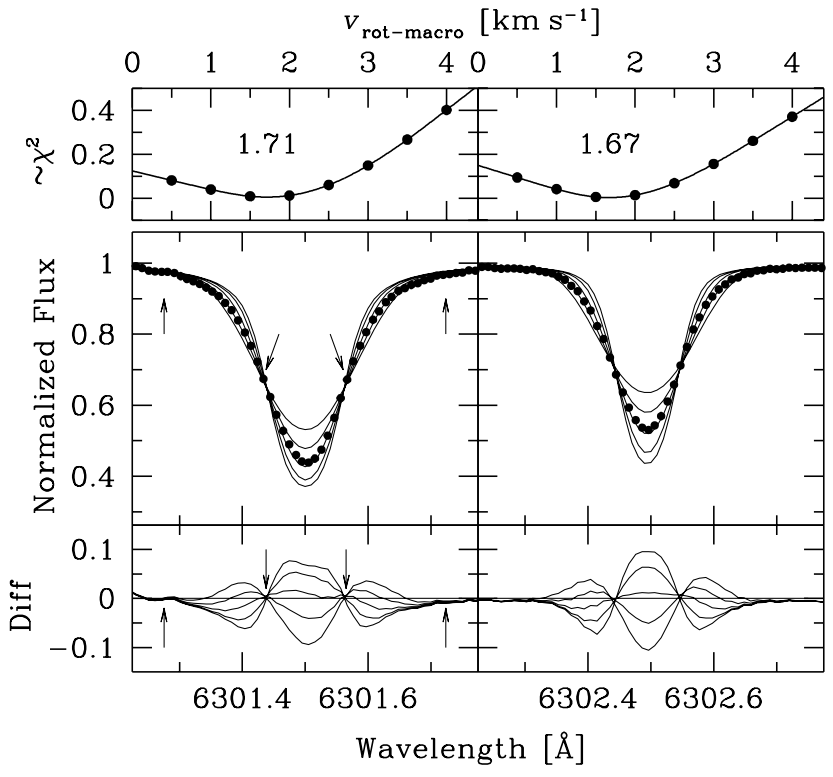

Fig. 4. Determination of $v_{\text {rot-macro }}$ for Hip 75181. The two Fe I lines at $6301.501 \AA$ (left panels) and $6302.494 \AA$ (right panels) have been used. The top panels show the un-normalized $\chi^{2}$-values for different values of $v_{\text {rot-macro }}$ in steps of $0.5 \mathrm{~km} \mathrm{~s}^{-1}$ and a spline function connecting them. The $v_{\text {rot-macro }}$ for which the $\chi^{2}$-functions are minimized are also indicated. The middle panels show the observed spectra (solid circles) and synthetic spectra for five different values of $v_{\text {rot-macro }}$ $\left(0.5,1,1.5,2,2.5 \mathrm{~km} \mathrm{~s}^{-1}\right)$. The bottom panels show the difference between the observed and the synthetic spectra in the middle panels. Uparrows indicate where the residuals converge, and down-arrows where they intersect.

order to make the shape of the synthetic line profiles similar to the observed lines. This was done since the Fe abundances that were used in the models have been determined from $\sim 150$ other, generally weaker, Fe I lines (see Bensby et al. 2003). We created 10 synthetic spectra for each star with $v_{\text {rot-macro }}$ ranging from 0.5 to $5 \mathrm{~km} \mathrm{~s}^{-1}$ in steps of $0.5 \mathrm{~km} \mathrm{~s}^{-1}$. For each $v_{\text {rot-macro }}$ we estimated the (un-normalized) $\chi^{2}$-value (i.e. the added sum of squares of the difference between observed and synthetic spectra in a certain wavelength interval).

When satisfactory $\log g f$-values have been reached the intersection points (indicated by down-arrows in Fig. 4) of the residuals between observed and synthetic spectra should lie on a vertical position equivalent to the level farther from the line core (i.e. where one approaches the continuum) and the residuals for the different $v_{\text {rot-macro }}$ converge (indicated by up-arrows in Fig. 4).

Next, the $\chi^{2}$-functions between observed and synthetic spectra were minimized in order to find the $v_{\text {rot-macro }}$ (see top panels in Fig. 4), using the new $\log g f$-values. We adopted the average $v_{\text {rot-macro }}$ as derived from the two Fe lines whenever possible (sometimes one of the two was affected by telluric lines). These values are listed in Col. 7 in Table 1.

\subsection{Solar analysis}

Our solar analysis is based on integrated solar light spectra that were obtained by observing the Moon, Jupiter's moon
Table 3. Our derived Solar oxygen abundances, given on the absolute abundance scale $\left(\epsilon(\mathrm{O})=\log N_{\mathrm{O}} / N_{\mathrm{H}}+12.0\right)$. All abundances have been determined under the assumption of LTE. NLTE corrections are done according to the prescription in Gratton et al. (2000). In the third column we give the O I triplet abundances corrected for NLTE. The two last columns give the differences between abundances from this work and the standard solar photospheric value, $\epsilon(\mathrm{O})_{\odot}=8.83$, from Grevesse \& Sauval (1998).

\begin{tabular}{ccccc}
\hline \hline Wavelength & $\epsilon(\mathrm{O})_{\odot}$ & \multicolumn{2}{c}{$\epsilon(\mathrm{O})_{\odot}$} & \multicolumn{2}{c}{ Difference } \\
$(\AA)$ & LTE & NLTE-corr & LTE & NLTE \\
\hline 6300.304 & 8.71 & & -0.12 & \\
6363.776 & 9.06 & & +0.23 & \\
7771.944 & 8.83 & 8.78 & \pm 0.00 & -0.05 \\
7774.166 & 8.88 & 8.83 & +0.05 & \pm 0.00 \\
7775.388 & 8.82 & 8.77 & -0.01 & -0.06 \\
\hline
\end{tabular}

Ganymede, and the afternoon sky with CES. The solar UVES spectrum is the Moon spectrum that can be obtained from ESO's web-pages ${ }^{5}$ and the solar FEROS spectra were obtained by observing the afternoon sky. The atmospheric parameters for our solar model are listed in Table 1 (see also Bensby et al. 2003 for further discussion).

Table 3 lists the abundances we derive for the Sun from the different lines using the methods that are described in more detail in Sects. 4.4, 4.5, and 4.6 below. For the O I triplet we also give NLTE corrected abundances (see Sect. 4.6). There are quite large differences between the abundances that the different indicators give. The [O I] lines give $\epsilon(\mathrm{O})_{6300}=8.71$ and $\epsilon(\mathrm{O})_{6363}=9.06$ while the $\mathrm{O}$ I triplet lines give abundances slightly higher than $8.8 \mathrm{dex}$ if no NLTE correction is applied and slightly lower than $8.8 \mathrm{dex}$ if corrected for NLTE effects according to the prescription of Gratton et al. (1999). That the abundances from the OI triplet do not agree with the abundances from the [O I] lines is not unexpected due to the NLTE effects that the triplet lines are known to suffer from (e.g. Kiselman 1991).

Lambert (1978) noted that there is a possible weak CN blend in the $[\mathrm{OI}]_{6363}$ line and estimated its equivalent width to be $\sim 0.18 \mathrm{~m} \AA$ in the Sun. Reducing our measured equivalent width by this amount results in an oxygen abundance that is 0.03 dex lower, i.e. there is still almost a 0.3 dex difference between abundances from the two forbidden lines. In order to bring down the abundance from the $\left[\mathrm{O} \mathrm{I}_{6363}\right.$ to what we find for the $[\mathrm{OI}]_{6300}$ line we would have to reduce the equivalent width from $2.3 \mathrm{~m} \AA$ to $1.0 \mathrm{~m} \AA$, i.e. the $\mathrm{CN}$ blend would have a strength of $1.3 \mathrm{~m} \AA$ in the Sun (if this is the only blend). Another possible reason for the inconsistency between the abundances from the two forbidden lines is erroneous $\log g f$-values. However, the $\log g f$-values for these lines come from the same calculations (see Sect. 3). If they are erroneous it is therefore most likely that both lines show deviations in the same direction and of equal size. Further, the $[\mathrm{OI}]_{6363}$ line is located in the wing of a Ca I auto-ionization line (Mitchell \& Mohler 1965) which

\footnotetext{
5 http://www . eso.org/observing/dfo/quality/UVES/ pipeline/solar_spectrum.html
} 


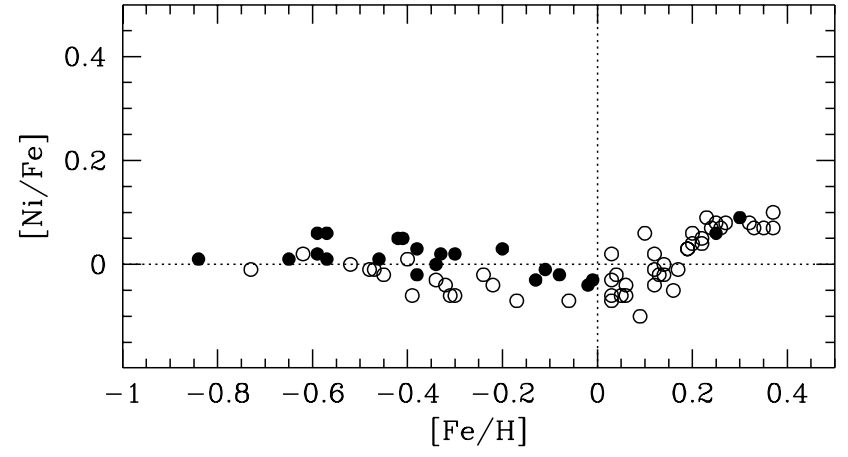

Fig. 5. The observed Ni trend for our stellar sample (see Bensby et al. 2003). The six additional stars have been included as well (Bensby et al., in prep.). Thin and thick disk stars are marked by open and filled circles, respectively.

might affect the strength, and thereby the derived oxygen abundance, from the $[\mathrm{OI}]_{6363}$ line (see Sect. 4.5).

The standard solar photospheric oxygen abundance is $\epsilon(\mathrm{O})_{\odot}=8.83 \pm 0.06$ (Grevesse \& Sauval 1998), However, depending on which spectral line that is used likely values range from $\sim 8.6$ dex to $\sim 8.9$ dex using one-dimensional (1D) models (Asplund 2003). The new 3D models give better agreement between the derived Solar abundance from different indicators (Asplund 2003). It should be noted that our solar oxygen abundance derived from the $[\mathrm{OI}]_{6300}$ line, using standard 1D LTE models, agrees nicely with what Allende Prieto et al. (2001) found using 3D models $\left(\epsilon(\mathrm{O})_{\odot}=8.69\right)$.

Since we want to compare the oxygen abundance trends to those oxygen trends that can be found in other works we normalize our abundances individually, line by line, to the solar values that we derived. This is done by correcting the abundances for all stars by subtracting the amounts that are given in the two final columns in Table 3. This means that the Sun will have $[\mathrm{O} / \mathrm{H}]=0$ independent of which line that is considered. It is these corrected oxygen abundances that we give in Table 1.

\subsection{Abundances from the $O \mathrm{I}$ line at $6300 \AA$}

The oxygen abundance from the $[\mathrm{OI}]_{6300}$ line has been determined for 63 of the 72 stars. In 60 cases we used CES spectra, in 2 cases FEROS spectra, and in 1 case a UVES spectrum, as indicated in Table 1. For 7 stars we were unable to use the $[\mathrm{OI}]_{6300}$ line due to strong interference from the telluric emission line (see Sect. 2.3). In two other cases where abundances from this line are lacking we did not have CES spectra and the lower quality of the FEROS spectra did not allow any abundance determination from this line.

The synthesis of the $[\mathrm{OI}]_{6300}$ line was done taking the blending NiI lines into account. Figure 5 shows the observed $\mathrm{Ni}$ trend for the stellar sample. It is obvious that $[\mathrm{Ni} / \mathrm{H}]$ is not varying in lockstep with $[\mathrm{Fe} / \mathrm{H}]$ which is commonly assumed in the modeling of the $[\mathrm{OI}]_{6300}$ line (e.g. Nissen et al. 2002). At $[\mathrm{Fe} / \mathrm{H}] \gtrsim 0$ solar-scaled $\mathrm{Ni}$ abundances will underestimate the $\mathrm{Ni}$ contribution to the line and consequently overestimate the final oxygen abundance. However, at $[\mathrm{Fe} / \mathrm{H}] \lesssim 0$ the $[\mathrm{Ni} / \mathrm{Fe}] \approx 0$ assumption might be reasonably correct as the Ni contribution to the total line strength decreases

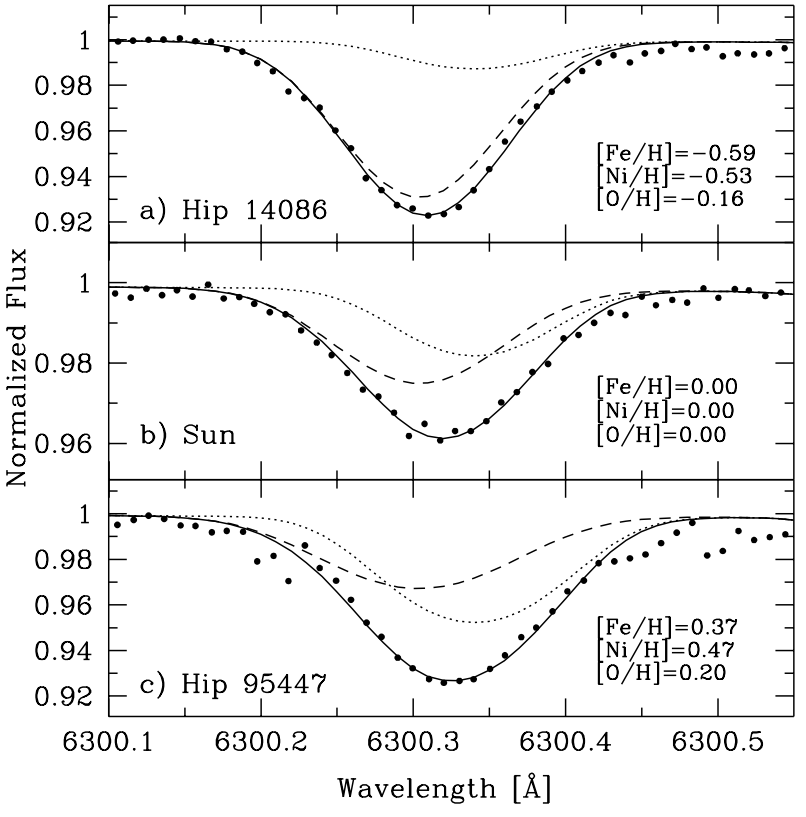

Fig. 6. Synthesis of the $[\mathrm{OI}]_{6300}$ line for three stars. In each plot the observed spectrum is marked by solid circles and the synthetic spectra by lines: the $[\mathrm{OI}]$ contribution by a dashed line; the contribution from the Ni lines by a dotted line; and the joint [O I]-Ni contribution by a solid line. The solar spectrum is in this case a Moon spectrum. Abundances as indicated.

rapidly below $[\mathrm{Fe} / \mathrm{H}]=0$. As can be seen in Fig. 6a the Ni I contribution to the combined line profile is small but not negligible for Hip 14086 which is a star that have $[\mathrm{Fe} / \mathrm{H}]=-0.59$. For the Sun the NiI contribution to the total absorption is slightly less than 50\% (Fig. 6b) and for Hip 95447 that has $[\mathrm{Fe} / \mathrm{H}]=0.37$ the Ni I contribution exceeds $50 \%$ of the combined [O I]-Ni I line profile (Fig. 6c).

To determine the oxygen abundance we first created a set of synthetic spectra representative of different oxygen abundances (in steps of $0.03 \mathrm{dex}$ ), using the $v_{\text {rot-macro }}$ determined in Sect. 4.2. Each synthetic spectrum was compared to the observed spectrum and a corresponding (un-normalized) $\chi^{2}$-value was calculated. In this way we got a $\chi^{2}$-function versus oxygen abundance that was minimized to find the best value of the oxygen abundance for each star. The corresponding abundance for the NiI blends were kept at a fixed value and was gathered from Bensby et al. (2003). Tables 1 and 4 lists the derived abundances.

\subsection{Abundances from the OI line at $6363 \AA$}

The $[\mathrm{OI}]_{6363}$ line is approximately $50 \%$ weaker than the $[\mathrm{O} \mathrm{I}]_{6300}$ line and is located in the right wing of a wide Ca I auto-ionization line (Mitchell \& Mohler 1965). This makes it difficult to synthesize this line well in our FEROS spectra. Instead we determined abundances from equivalent width measurements. That the continuum is depressed is then of small concern since the local continuum can be set at every measurement. However, depending on at which depth in the stellar atmosphere this Ca I auto-ionization line forms its effect on the equivalent width of the $[\mathrm{OI}]_{6363}$ line will vary. If it is formed 

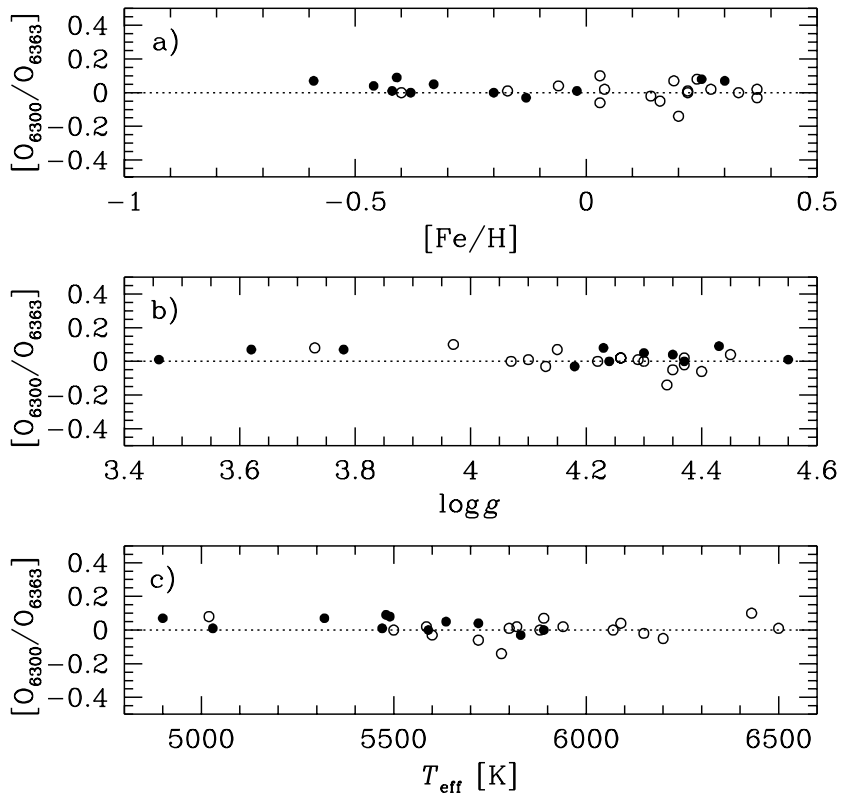

Fig. 7. Difference between the oxygen abundances from the $[\mathrm{O} \mathrm{I}]_{6300}$ line and the $[\mathrm{OI}]_{6363}$ line versus $[\mathrm{Fe} / \mathrm{H}], \log g$, and $T_{\text {eff }}$. Thin and thick disk stars are marked by open and filled circles, respectively.

below the $[\mathrm{O}]_{6363}$ line it will only contribute to the continuum flux, while if it forms higher up in the atmosphere than the $[\mathrm{OI}]_{6363}$ line it will increase the strength of the $[\mathrm{O}]_{6363}$ line. If this latter is the case and if the estimation of the strength of the CN blend by Lambert (1978) is correct (and also assuming that $\mathrm{CN}$ is the only blend), this implies that the $\mathrm{Ca}$ I auto-ionization line contributes $\sim 1.1 \mathrm{~m} \AA$ to the $[\mathrm{O} \mathrm{I}]_{6363}$ line in the Sun.

Both $[\mathrm{C} / \mathrm{H}]$ and $[\mathrm{N} / \mathrm{H}]$ evolves in lockstep with $[\mathrm{Fe} / \mathrm{H}]$ at high metallicities (Andersson \& Edvardsson 1994; Gustafsson et al. 1999; Clegg et al. 1981; Shi et al. 2002). From this it does not necessarily follow that $[\mathrm{CN} / \mathrm{H}]$ evolves like $[\mathrm{Fe} / \mathrm{H}]$. The amount of $\mathrm{CN}$ present in a stellar atmosphere depends on its dissociation energy and the temperature in the stellar atmosphere. The dissociation energy for $\mathrm{CN}$ is, however, not well known (see discussion in de Laverny \& Gustafsson 1998). But, as can be seen in Figs. $7 \mathrm{a}$, c, there is no trends in $\left[\mathrm{O}_{6300} / \mathrm{O}_{6363}\right]$ with either $[\mathrm{Fe} / \mathrm{H}]$ or $T_{\text {eff }}$ which possibly indicates that $[\mathrm{CN} / \mathrm{H}]$ evolves with $[\mathrm{Fe} / \mathrm{H}]$ in our stars.

Since we have neglected the CN blend (and other potential effects) in the abundance determination there is a potential risk that the oxygen abundances from the $[\mathrm{O} \mathrm{I}]_{6363}$ line are too high. How it will affect the $[\mathrm{O} / \mathrm{Fe}]$ vs. $[\mathrm{Fe} / \mathrm{H}]$ trends depends on how the $\mathrm{CN}$ abundance evolves with $[\mathrm{Fe} / \mathrm{H}]$. It seems that $[\mathrm{CN} / \mathrm{Fe}] \approx 0$ for all $[\mathrm{Fe} / \mathrm{H}]$ (if $\mathrm{CN}$ is the only blend) since the (normalized) oxygen abundances are the same for both of the forbidden lines (see Figs. 7a-c). The effect on the derived $[\mathrm{O} / \mathrm{Fe}]$ trends will therefore only be a systematic vertical shift for all stars since we neglected the contribution from the blend in our Solar analysis as well.

Due to the lower resolution and the lower signal-to-noise of the FEROS spectra compared to the CES spectra we could only measure the equivalent width of the $[\mathrm{OI}]_{6363}$ line in 32 stars.
A UVES spectrum was used in one case (see Table 1). The measured equivalent widths for the $[\mathrm{OI}]_{6363}$ line are listed in Table 4.

\subsection{Abundances from the OI triplet at $7774 \AA$}

Abundances were determined through equivalent width measurements of the permitted $\mathrm{O}_{\mathrm{I}}$ lines at $7771.95 / 7774.17 / 7775.39 \AA$ in our FEROS spectra for 69 stars. The UVES spectra for the three additional stars could not be used since the triplet lines are not present due to the setting of the spectrograph.

The triplet lines are known to give discrepant abundances under the assumption of LTE compared to the $[\mathrm{OI}]_{6300}$ line (e.g. Eriksson \& Toft 1979; Kiselman 1991, 1993; Gratton et al. 1999). An LTE analysis will always give abundances that are overestimated. In our analysis we correct the derived abundances for NLTE effects according to the prescription of Gratton et al. (1999). These non-LTE corrections are based on comparisons between abundances obtained with statistical equilibrium calculations and abundances provided by an LTE analysis with the Uppsala MARCS models.

Table 4 lists our measured equivalent widths as well as abundances for individual lines and NLTE corrections from Gratton et al. (1999). Table 1 gives the mean abundances (both LTE and NLTE) from the three lines.

In Figs. 8a-c we compare the abundances, not corrected for NLTE effects (but normalized to the Sun), from the triplet lines to those from the $[\mathrm{OI}]_{6300}$ line for the 60 stars that have both the $[\mathrm{OI}]_{6300}$ and the triplet lines analyzed (compare Table 1). In summary, abundances from the triplet give higher abundances than what the forbidden line gives. The average difference and the standard deviation are $\left\langle\left[\mathrm{O}_{6300} / \mathrm{O}_{7774}\right]\right\rangle=-0.049 \pm$ 0.084 . The largest differences are seen at the highest metallicities $([\mathrm{Fe} / \mathrm{H}] \gtrsim 0)$ and highest temperatures $\left(T_{\text {eff }} \gtrsim 6100 \mathrm{~K}\right)$ and, possibly, for $\log g \gtrsim 4.4$. For the NLTE corrected abundances the average difference and the standard deviation is lower $\left\langle\left[\mathrm{O}_{6300} / \mathrm{O}_{7774}\right]\right\rangle=-0.015 \pm 0.077$ (see Figs. $8 \mathrm{~d}-\mathrm{f}$ ). The trends with $[\mathrm{Fe} / \mathrm{H}]$ and $\log g$ are still present but somewhat less prominent and the trend with $T_{\text {eff }}$ has changed significantly. The triplet lines now give increasingly lower abundances when going to higher $T_{\text {eff }}$. The NLTE corrections of Gratton et al. (1999) therefore could be somewhat overestimated for the parameter space that our stars span.

A weighted linear regression ${ }^{6}$ with $\log T_{\text {eff }}, \log g$, and $[\mathrm{Fe} / \mathrm{H}]$ as the dependent variables and the difference between the oxygen abundances from the $[\mathrm{OI}]_{6300}$ line and the triplet lines $\left(\left[\mathrm{O}_{6300} / \mathrm{O}_{7774}\right]\right)$ as the independent gives:

$$
\begin{aligned}
\Delta_{\mathrm{NLTE}}^{7774} \equiv\left[\mathrm{O}_{6300} / \mathrm{O}_{7774}\right]= & -0.046( \pm 0.02) \\
& -0.89( \pm 0.37) \cdot \log \left(T_{\mathrm{eff}} / T_{\mathrm{eff}, \odot}\right) \\
& +0.10( \pm 0.04) \cdot \log \left(g / g_{\odot}\right) \\
& -0.10( \pm 0.03) \cdot[\mathrm{Fe} / \mathrm{H}] .
\end{aligned}
$$

${ }^{6}$ For a description of the algorithm see the manual entry for the "robustfit" function in the MATLAB Statistics Toolbox User's Guide (www.mathworks.com). 
Table 4. Oxygen abundances for the 72 program stars. Column 1 gives the Hipparcos number, Cols. 2-6 the derived oxygen abundances for the different indicators relative to the photospheric oxygen abundance $\left(\epsilon(\mathrm{O})_{\odot}=8.83\right)$ from Grevesse \& Sauval $(1998)$. Columns 7-11 give the abundances relative to our solar abundances, i.e. $[\mathrm{O} / \mathrm{H}]_{\odot}=0$ for all indicators. Columns $12-14$ give the abundances for the triplet that have been corrected for NLTE effects according the the prescription in Gratton et al. (2000), and normalized to the new NLTE corrected abundance for the Sun. Column 15 gives the NLTE correction terms from Gratton et al. (2000) (GCEG) that were applied to the abundances in Cols. 12-14. Column 15 gives the empirical NLTE correction we determined (Eqs. (1) and (2)). Columns 16-19 give the measured equivalent widths for the $[\mathrm{OI}]_{6363}$ and the triplet lines. The full table is available in electronic form at the CDS via anonymous ftp to cdsarc.u-strasbg.fr (130.79.128.5) or via http://cdsweb.u-strasbg.fr/cgi-bin/qcat?/A+A/415/155

\begin{tabular}{|c|c|c|c|c|c|c|c|c|c|c|c|c|c|c|c|c|c|c|c|}
\hline \multirow[t]{2}{*}{ Hip } & \multicolumn{5}{|c|}{$\longrightarrow[\mathrm{O} / \mathrm{H}]-$} & \multicolumn{5}{|c|}{$-[\mathrm{O} / \mathrm{H}]_{\text {norm }}$} & \multicolumn{3}{|c|}{$-[\mathrm{O} / \mathrm{H}]_{\mathrm{norm}}^{\mathrm{NLTE}} \longrightarrow$} & \multicolumn{2}{|c|}{ NLTE-corr. } & \multicolumn{4}{|c|}{$\longrightarrow \mathrm{EW}[\mathrm{m \AA}] \longrightarrow$} \\
\hline & 6300 & 6363 & 7772 & 7774 & 7775 & 6300 & 6363 & 7772 & 7774 & 7775 & 7772 & 7774 & 7775 & GCEG & TW & 6363 & 7772 & 7774 & 7775 \\
\hline Sun & -0.12 & 0.23 & 0.00 & 0.05 & -0.01 & 0.00 & 0.00 & 0.00 & 0.00 & 0.00 & 0.00 & 0.00 & 0.00 & -0.05 & -0.04 & 2.3 & 68.3 & 61.9 & 46.4 \\
\hline 3142 & -0.37 & & -0.15 & -0.16 & -0.22 & -0.25 & & -0.15 & -0.21 & -0.21 & -0.22 & -0.28 & -0.28 & -0.12 & -0.06 & & 88.3 & 77.1 & 58.6 \\
\hline 3170 & & & -0.09 & -0.07 & 0.02 & & & -0.09 & -0.12 & 0.03 & -0.13 & -0.16 & -0.01 & -0.09 & -0.06 & & 83.2 & 74.5 & 65.8 \\
\hline : & : & : & $\vdots$ & & & : & & . & & : & & : & & . & . & & . & : & \\
\hline
\end{tabular}
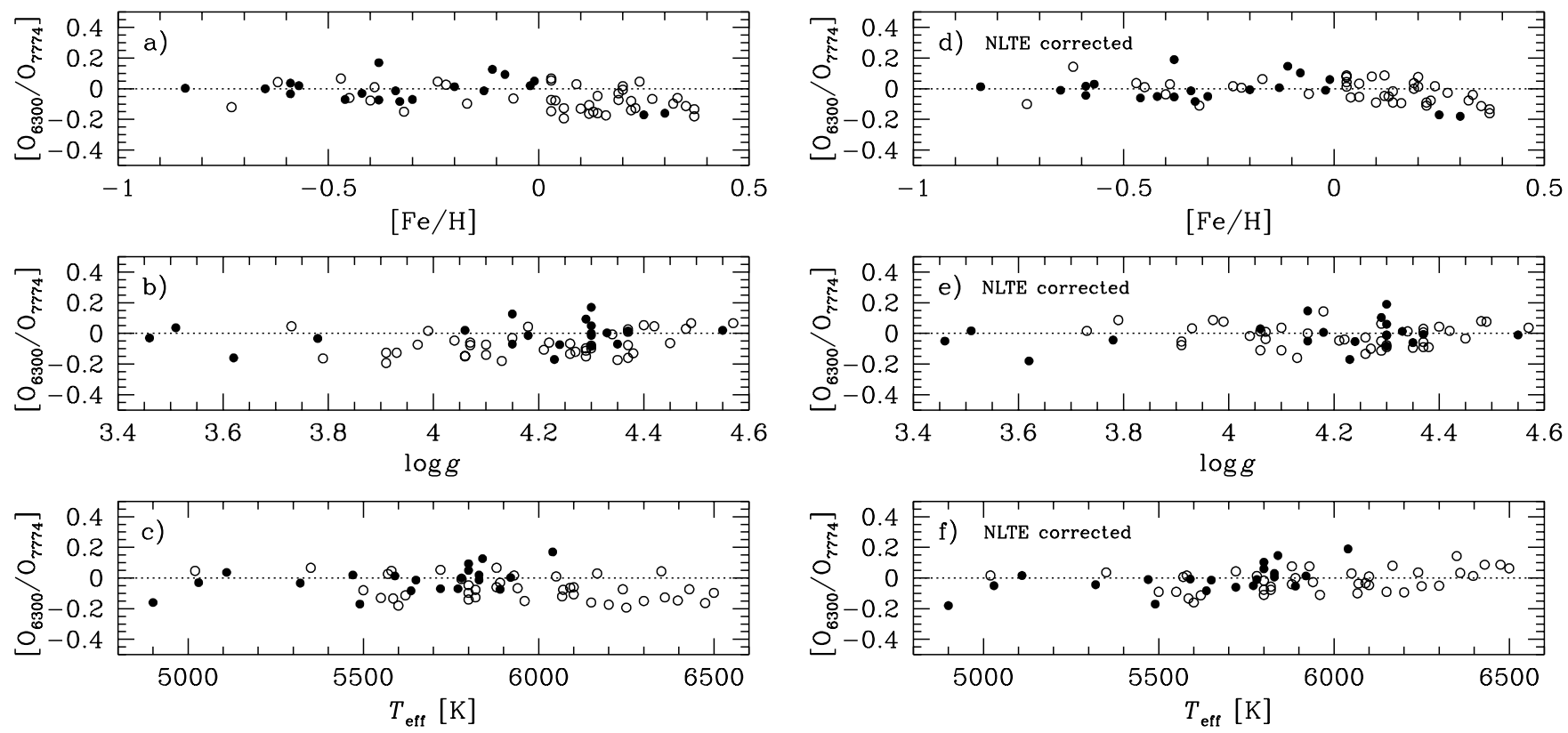

Fig. 8. a)-c) Difference between the oxygen abundances from the $[\mathrm{OI}]_{6300}$ line and the O I triplet at $7774 \AA$ i versus $[\mathrm{Fe} / \mathrm{H}], \log g$, and $T_{\text {eff }}$. No NLTE corrections have been applied to the abundances from the triplet. d)-f) Difference between the oxygen abundances from the [O I $]_{6300}$ line and the NLTE corrected abundances (according to the prescription in Gratton et al. 1999) from the O I triplet at $7774 \AA$ versus [Fe/H], log $g$, and $T_{\text {eff }}$. Thin and thick disk stars are marked by open and filled circles, respectively.

This empirical correction can then be applied to the abundances from the O I triplet:

$[\mathrm{O} / \mathrm{H}]_{\mathrm{NLTE}}^{7774}=[\mathrm{O} / \mathrm{H}]_{\mathrm{LTE}}^{7774}+\Delta_{\mathrm{NLTE}}^{7774}$.

The derived relationship is valid for stars with: $5000 \lesssim T_{\text {eff }} \lesssim 6500,3.5 \lesssim \log g \lesssim 4.5$, and $-1 \lesssim[\mathrm{Fe} / \mathrm{H}] \lesssim 0.5$.

\section{Errors in resulting abundances}

The random (internal) errors in the derived oxygen abundances can have several causes. Effective temperature, surface gravity, microturbulence, measured equivalent widths, and atomic data are the main contributors. In estimating the total error we assume that the errors arising from each of the sources are independent. The error due to each parameter is estimated by varying the parameter in question.
As in Bensby et al. (2003) we estimate the effects of the uncertainties in these parameters on the derived oxygen abundances by increasing $T_{\text {eff }}$ by $70 \mathrm{~K}, \log g$ by $0.1 \mathrm{dex}$, microturbulence $\left(\xi_{\mathrm{t}}\right)$ by $0.15 \mathrm{~km} \mathrm{~s}^{-1},[\mathrm{Fe} / \mathrm{H}]$ by $0.1 \mathrm{dex}$, correction factor $\left(\delta \gamma_{6}\right)$ to the classic Unsöld by $50 \%$, and the measured equivalent widths by $5-10 \%$. This uncertainty in the measured equivalent widths correspond to an uncertainty in the derived abundances of 0.02-0.04 dex.

If the abundance is derived from $N$ spectral lines the uncertainty should be reduced by a factor $N^{1 / 2}$ to give the formal error in the mean. For the $[\mathrm{OI}]_{6363}$ line we adopt the higher value $(0.04 \mathrm{dex})$ since the line is very weak and difficult to measure. For the OI triplet abundance we adopt the lower value $(0.02 / \sqrt{3} \mathrm{dex})$ since the three lines are strong and easy to measure. 
Table 5. Estimates of the effects on the derived abundances due to internal (random) errors for three stars. When calculating $\Delta W_{\lambda} / \sqrt{N}$ for the Fe abundances we have assumed $\Delta W_{\lambda}=5 \%$ for Hip 88622 and Hip 82588 and $\Delta W_{\lambda}=10 \%$ for Hip 103682 (see Bensby et al. 2003). For the $[\mathrm{OI}]_{6363}$ abundances we adopted $\Delta W_{\lambda}=10 \%$ and for the O I triplet abundances $\Delta W_{\lambda}=5 \%$ for all three stars. The total random uncertainties $\left(\sigma_{\text {rand }}\right)$ were calculated assuming the individual errors to be uncorrelated. The final line gives the average of the total random uncertainties for the three stars.

\begin{tabular}{|c|c|c|c|c|c|c|c|}
\hline & & -6 & $00-$ & -6 & $63-$ & -77 & $74-$ \\
\hline & {$\left[\frac{\mathrm{FeI}}{\mathrm{H}}\right]$} & {$\left[\frac{\mathrm{O}_{\mathrm{I}}}{\mathrm{H}}\right]$} & {$\left[\frac{\mathrm{O}_{\mathrm{I}}}{\mathrm{Fe}}\right]$} & {$\left[\frac{\mathrm{O}_{1}}{\mathrm{H}}\right]$} & {$\left[\frac{\mathrm{O}_{\mathrm{I}}}{\mathrm{Fe}}\right]$} & {$\left[\frac{\mathrm{O}_{\mathrm{I}}}{\mathrm{H}}\right]$} & {$\left[\frac{\mathrm{O}_{\mathrm{I}}}{\mathrm{Fe}}\right]$} \\
\hline & \multicolumn{7}{|c|}{ Hip 88622} \\
\hline$\Delta T_{\mathrm{eff}}=+70 \mathrm{~K}$ & 0.06 & 0.02 & -0.04 & 0.02 & -0.04 & -0.06 & -0.12 \\
\hline$\Delta \log g=+0.1$ & -0.01 & 0.04 & 0.05 & 0.05 & 0.06 & 0.02 & 0.01 \\
\hline$\Delta \xi_{\mathrm{t}}=+0.15 \mathrm{~km} \mathrm{~s}^{-1}$ & -0.02 & 0.00 & 0.02 & 0.00 & 0.02 & -0.01 & 0.01 \\
\hline$\Delta[\mathrm{Fe} / \mathrm{H}]=+0.1$ & 0.01 & 0.03 & 0.02 & 0.03 & 0.02 & 0.01 & 0.00 \\
\hline$\Delta \delta \gamma_{6}=+50 \%$ & 0.00 & 0.00 & 0.00 & 0.00 & 0.00 & 0.00 & 0.00 \\
\hline$\Delta W_{\lambda} / \sqrt{N}$ & 0.00 & & & 0.04 & 0.04 & 0.01 & 0.01 \\
\hline$\sigma_{\text {form }}(\mathrm{Ni})=0.09 / \sqrt{49}$ & & 0.00 & 0.00 & & & & \\
\hline \multirow[t]{2}{*}{$\sigma_{\text {rand }}$} & 0.06 & 0.05 & 0.07 & 0.07 & 0.09 & 0.07 & 0.12 \\
\hline & \multicolumn{7}{|c|}{ Hip 82588} \\
\hline$\Delta T_{\text {eff }}=+70 \mathrm{~K}$ & 0.05 & 0.01 & -0.04 & 0.01 & -0.04 & -0.08 & -0.13 \\
\hline$\Delta \log g=+0.1$ & -0.01 & 0.04 & 0.05 & 0.05 & 0.06 & 0.02 & 0.03 \\
\hline$\Delta \xi_{\mathrm{t}}=+0.15 \mathrm{~km} \mathrm{~s}^{-1}$ & -0.03 & 0.00 & 0.03 & 0.00 & 0.03 & -0.01 & 0.02 \\
\hline$\Delta[\mathrm{Fe} / \mathrm{H}]=+0.1$ & 0.01 & 0.04 & 0.03 & 0.03 & 0.02 & 0.02 & 0.01 \\
\hline$\Delta \delta \gamma_{6}=+50 \%$ & -0.01 & 0.00 & 0.01 & 0.00 & 0.01 & 0.00 & 0.01 \\
\hline$\Delta W_{\lambda} / \sqrt{N}$ & 0.00 & & & 0.04 & 0.04 & 0.01 & 0.01 \\
\hline$\sigma_{\text {form }}(\mathrm{Ni})=0.08 / \sqrt{54}$ & & -0.01 & 0.01 & & & & \\
\hline \multirow[t]{2}{*}{$\sigma_{\text {rand }}$} & 0.06 & 0.06 & 0.08 & 0.07 & 0.08 & 0.09 & 0.13 \\
\hline & \multicolumn{7}{|c|}{ Hip 103682} \\
\hline$\Delta T_{\text {eff }}=+70 \mathrm{~K}$ & 0.05 & 0.02 & -0.03 & 0.00 & -0.05 & -0.06 & -0.11 \\
\hline$\Delta \log g=+0.1$ & -0.01 & 0.05 & 0.06 & 0.04 & 0.05 & 0.02 & 0.03 \\
\hline$\Delta \xi_{\mathrm{t}}=+0.15 \mathrm{~km} \mathrm{~s}^{-1}$ & -0.05 & 0.00 & 0.05 & 0.00 & 0.05 & -0.01 & 0.04 \\
\hline$\Delta[\mathrm{Fe} / \mathrm{H}]=+0.1$ & 0.00 & 0.03 & 0.03 & 0.03 & 0.03 & 0.01 & 0.01 \\
\hline$\Delta \delta \gamma_{6}=+50 \%$ & 0.00 & 0.00 & 0.00 & 0.00 & 0.00 & 0.00 & 0.00 \\
\hline$\Delta W_{\lambda} / \sqrt{N}$ & 0.00 & & & 0.04 & 0.04 & 0.01 & 0.01 \\
\hline$\sigma_{\text {form }}(\mathrm{Ni})=0.11 / \sqrt{54}$ & & -0.01 & 0.01 & & & & \\
\hline$\sigma_{\text {rand }}$ & 0.07 & 0.06 & 0.09 & 0.06 & 0.10 & 0.07 & 0.12 \\
\hline$\left\langle\sigma_{\text {rand }}\right\rangle$ & 0.06 & 0.06 & 0.08 & 0.07 & 0.09 & 0.08 & 0.12 \\
\hline
\end{tabular}

The uncertainty in the abundance from the $[\mathrm{O}]_{6300}$ line is also dependent on the accuracy of the derived $\mathrm{Ni}$ abundance. From the discussion in Sect. 4.4 it is also clear that stars at high $[\mathrm{Fe} / \mathrm{H}]$ are more affected by this than those at low $[\mathrm{Fe} / \mathrm{H}]$. To estimate these uncertainties we vary the $\mathrm{Ni}$ abundance according to the observed formal error in the mean of the Ni abundance (see Bensby et al. 2003) and then derive new oxygen abundances from line synthesis.

These error estimates are exemplified for three stars and are listed in Table 5. It should be noted that the error estimates for the damping constants only apply to those lines marked by a " $U$ " in Table 2 . The total internal (random) errors are typically 0.06 dex in $[\mathrm{O} / \mathrm{H}]_{6300}$ and $[\mathrm{O} / \mathrm{H}]_{6363}$, and 0.08 dex in $[\mathrm{O} / \mathrm{H}]_{7774}$.

We note that the oxygen trends in Figs. $9 \mathrm{a}$ and $\mathrm{b}$ are very tight and that the two populations separate very well. That this separation should be so distinct and the internal errors as large as the ones estimated in this section seems unlikely.
We therefore conclude that our error estimates in Table 5 are upper limits to the internal errors.

\section{6. $[\mathrm{O} / \mathrm{Fe}]$ vs. $[\mathrm{Fe} / \mathrm{H}]$ trends}

Our oxygen trends derived from the $[\mathrm{OI}]_{6300}$ line are shown in Fig. 9a. The thin and thick disk trends are well separated and distinct. At $[\mathrm{Fe} / \mathrm{H}]<-0.4$ the $[\mathrm{O} / \mathrm{Fe}]$ trend is nearly flat for the thick disk stars, showing an oxygen overabundance of $[\mathrm{O} / \mathrm{Fe}] \approx 0.4$. Then, at $[\mathrm{Fe} / \mathrm{H}] \approx-0.4$, there is a downward trend towards solar values. This trend is in good agreement with what we found for other $\alpha$-elements ( $\mathrm{Mg}, \mathrm{Si}, \mathrm{Ca}$, and Ti) (see Bensby et al. 2003; Feltzing et al. 2003). As we already noted then, this turn-over or "knee" is most likely an indication of contribution from SN Ia to the chemical enrichment of the interstellar medium.

The thin disk $[\mathrm{O} / \mathrm{Fe}]$ trend is very well defined with a small internal scatter. It shows an overabundance of oxygen of $[\mathrm{O} / \mathrm{Fe}] \approx 0.3$ at $[\mathrm{Fe} / \mathrm{H}] \approx-0.7$ and continues linearly down to an underabundance of $[\mathrm{O} / \mathrm{Fe}] \approx-0.2$ at $[\mathrm{Fe} / \mathrm{H}] \approx 0.4$. Such a trend has previously only been seen by Castro et al. (1997) who analyzed the $[\mathrm{OI}]_{6300}$ line in seven stars with $+0.1 \lesssim[\mathrm{Fe} / \mathrm{H}] \lesssim+0.5$, and by Chen et al. (2003) who analyzed the OI triplet in 15 stars with $-0.1 \lesssim[\mathrm{Fe} / \mathrm{H}] \lesssim+0.5$. However, only seven stars in the Chen et al. (2003) study had $[\mathrm{Fe} / \mathrm{H}]>+0.1$.

Compared to the abundance trends obtained from the $[\mathrm{OI}]_{6300}$ line, Fig. $9 \mathrm{a}$, the trends based on the $[\mathrm{OI}]_{6363}$ line is practically identical even though the number of stars is considerably smaller. Since the $[\mathrm{O} \mathrm{I}]_{6363}$ line of a star is not affected by its $\mathrm{Ni}$ abundance this provides an independent test of our analysis of the $[\mathrm{OI}]_{6300}$ line. We therefore believe that the downward trend in $[\mathrm{O} / \mathrm{Fe}]$ that we see at $[\mathrm{Fe} / \mathrm{H}]>0$ as well as the separation of the thin and thick disk trends at $[\mathrm{Fe} / \mathrm{H}]<0$ are real.

In Fig. 9c we show the abundance trends derived from the O I triplet that have not been corrected for NLTE effects. At $[\mathrm{Fe} / \mathrm{H}]<0$ the trends for the thin and the thick disks are essentially the same as we saw from the forbidden lines (Figs. 9a, b) suggesting that the NLTE effects might not be so severe at these metallicities (see also Fig. 8a). However, the scatter is significantly larger, especially for the thin disk. At super-solar $[\mathrm{Fe} / \mathrm{H}]$ there is a large scatter and no clear sign of the downward trend that we see in the $[\mathrm{OI}]$ lines. The $[\mathrm{O} / \mathrm{Fe}]$ trend based on the NLTE corrected (according to the prescription by Gratton et al. 1999) O I abundances is shown in Fig. 9d. While not much have changed at $[\mathrm{Fe} / \mathrm{H}]<0$, except for a somewhat more well-defined thin disk trend, the scatter is considerably reduced at $[\mathrm{Fe} / \mathrm{H}]>0$ and with a marginal downward trend in $[\mathrm{O} / \mathrm{Fe}]$. However, the forbidden lines always give superiorly defined trends.

\section{Discussion}

In Fig. 10 we show the oxygen trends, both the common $[\mathrm{O} / \mathrm{Fe}]$ vs. $[\mathrm{Fe} / \mathrm{H}]$ trend and the nowadays often used $[\mathrm{Fe} / \mathrm{H}]$ vs. $[\mathrm{O} / \mathrm{H}]$ trend, with all 72 stars in the sample included. The abundances from the $[\mathrm{OI}]_{6300}$ line have been used whenever available. If not, our first choice are the abundances from the $[\mathrm{O} \text { I }]_{6363}$ line 

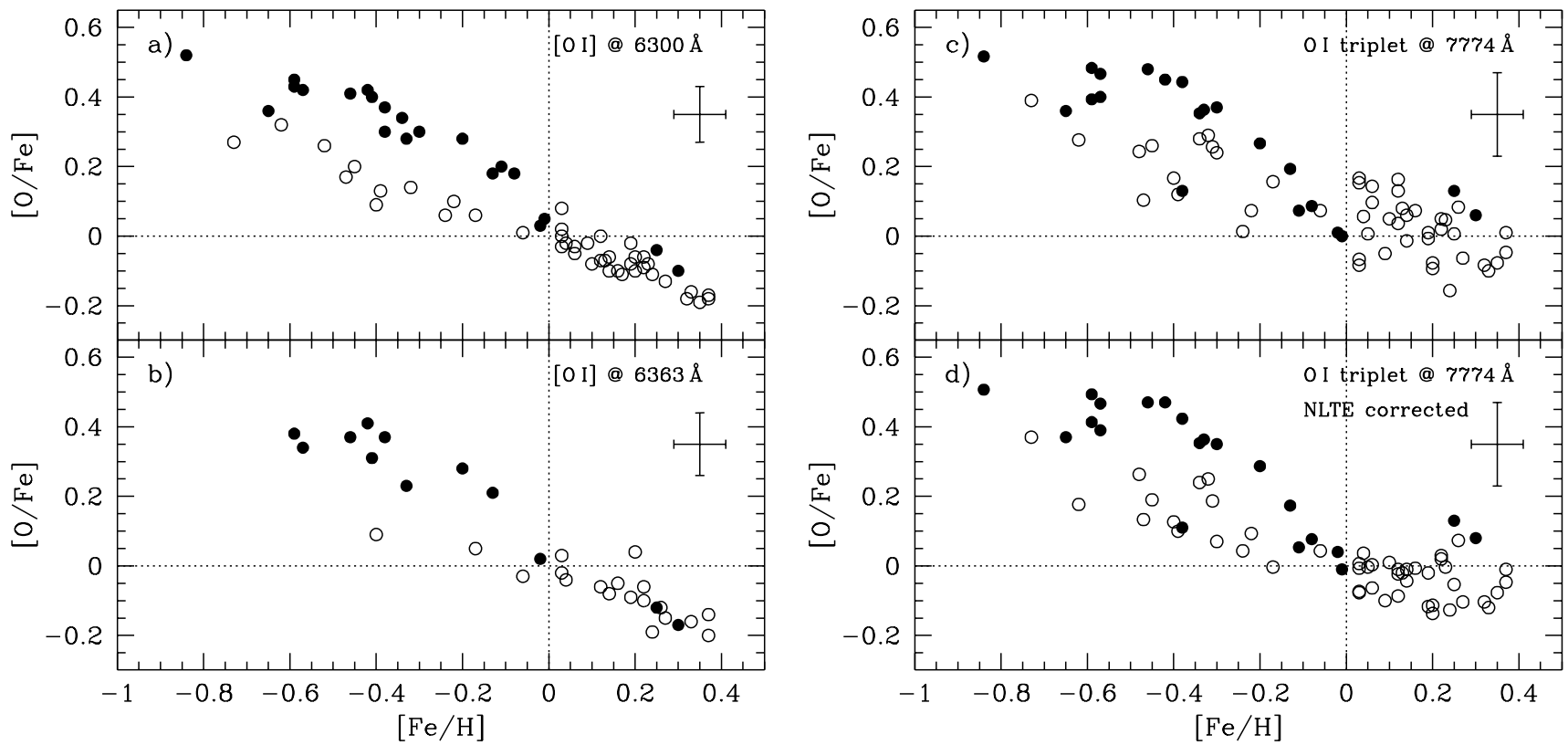

Fig. 9. The oxygen trends for our stellar sample as it appears when using; a) the $[\mathrm{O} \mathrm{I}]_{6300}$ line, b) the $[\mathrm{O} \text { I }]_{6363}$ line, c) the O I triplet at $7774 \AA$, and d) the O I triplet at $7774 \AA$ A corrected for NLTE effects according to the prescription in Gratton et al. (1999) (but see discussion in Sect. 4.6). Thin and thick disk stars are marked by open and filled circles, respectively. The error-bars in the upper right corner are the total random errors (see Sect. 5 and Table 5).
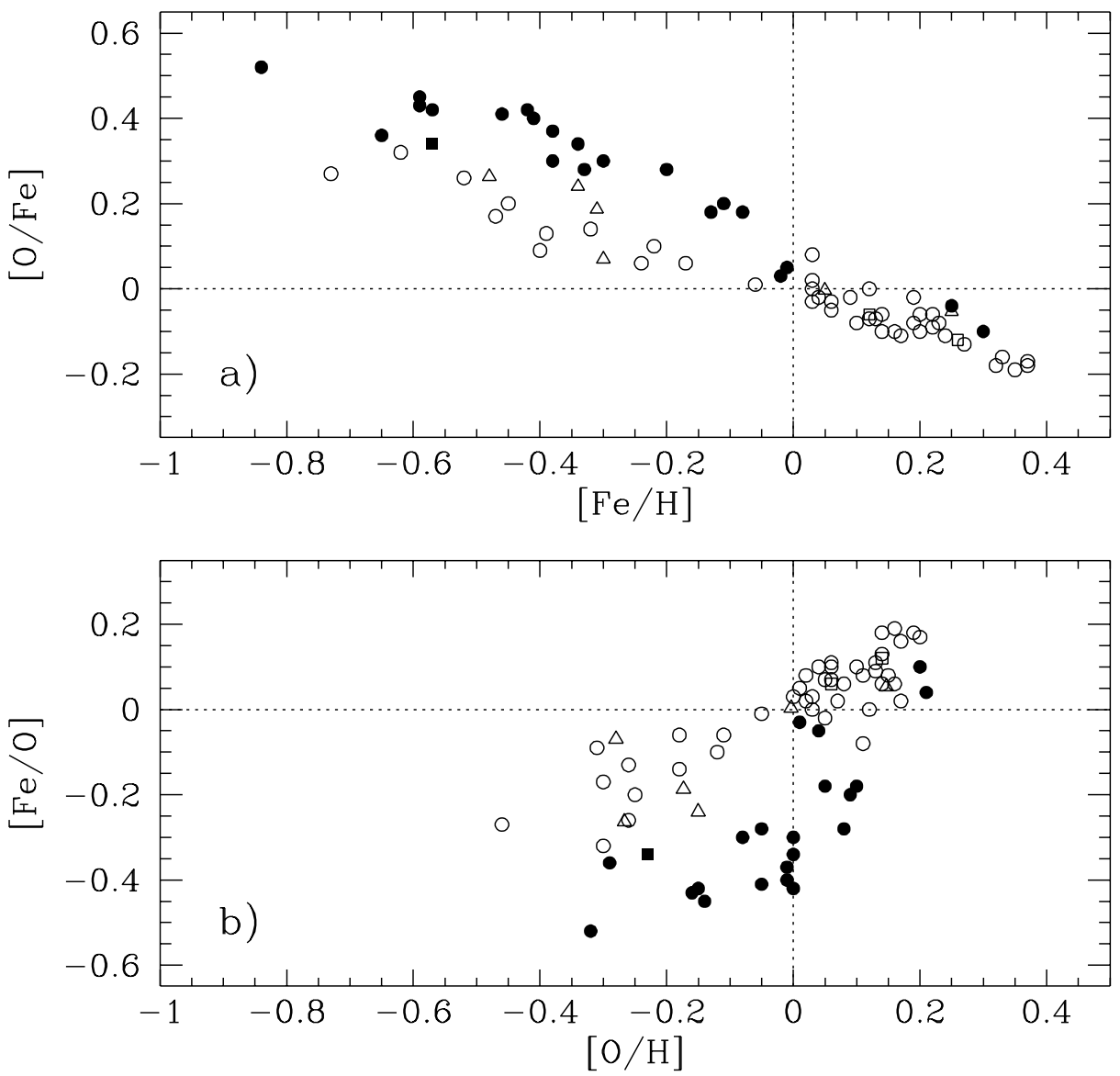

Fig. 10. The oxygen trend, as it appears when all 72 stars are included. a) shows the $[\mathrm{O} / \mathrm{Fe}]$ vs. $[\mathrm{Fe} / \mathrm{H}]$ trend and b) the $[\mathrm{Fe} / \mathrm{O}]$ vs. $[\mathrm{O} / \mathrm{H}]$ trend. Abundances derived from the $[\mathrm{OI}]_{6300}$ line are marked by circles, abundances from the $[\mathrm{OI}]_{6363}$ line by squares, and NLTE corrected (according to the prescription in Gratton et al. 2000) abundances from the O I triplet at $7774 \AA$ A by triangles. Thin and thick disk stars are marked by open and filled symbols, respectively. 
and second the NLTE corrected (according to the prescription of Gratton et al. 1999) abundances from the O I triplet.

\subsection{Interpretation of the oxygen trends in the thin and thick disks}

\subsubsection{Strengthening of the trends}

Nissen et al. (2002) analyzed oxygen in 41 stars. Out of those, 31 stars have kinematic data available. For these we have calculated their $T D / D$ and $T D / H$ ratios and selected those with $T D / D>1$ and $T D / H>1$ as thick disk stars and those with $T D / D<1$ and $T D / H>1$ as thin disk stars. Four stars that have $T D / D>1$ and $T D / H<1$ were consequently interpreted as halo stars. Apart from the 4 halo stars this left us with 10 thick disk and 17 thin disk stars from Nissen et al. (2002). In Fig. 11 we add these stars to our own data set.

The thick and thin disk trends seen in Fig. 10a are further strengthened by the inclusion of the stars from Nissen et al. (2002) (see Fig. 11). Especially so the flat trend in the thick disk up till $[\mathrm{Fe} / \mathrm{H}]=-0.5$ and the break in $[\mathrm{O} / \mathrm{Fe}]$ at $[\mathrm{Fe} / \mathrm{H}] \approx-0.4$. The thin disk trend at $[\mathrm{Fe} / \mathrm{H}] \lesssim 0$ is nicely supported too. Intriguingly, the most metal-rich halo star is low in $[\mathrm{O} / \mathrm{Fe}]$ compared to thick disk stars at the same metallicity, which was also found by Nissen \& Schuster (1997). They speculated that these anomalous halo stars can have been accreted from dwarf galaxies that have a different chemical histories than the Galactic disks.

For the metal-rich disk $([\mathrm{Fe} / \mathrm{H}]>0)$ the Nissen et al. (2002) stars have higher oxygen abundances than ours and level out to a constant value of $[\mathrm{O} / \mathrm{Fe}] \sim 0$. The Nissen et al. (2002) stars include a re-analysis of the Nissen \& Edvardsson (1992) data that did not account for the Ni blend in the $[\mathrm{OI}]_{6300}$ line. Nissen et al. (2002) calculated the equivalent width of the $\mathrm{Ni}$ blend assuming solar-scaled $\mathrm{Ni}$ abundances (i.e. $[\mathrm{Ni} / \mathrm{Fe}]=0$ ) for all metallicities (which is not consistent with what we see in our Ni trends, Fig. 5, but is consistent to earlier studies e.g. Edvardsson et al. 1993). Then they subtracted this calculated $\mathrm{Ni}$ contribution from the observed equivalent width of the [OI] line and then re-calculated the oxygen abundances. Apart from an absolute shift in the oxygen abundances, their new results showed essentially no differences compared to the abundance trends in Nissen \& Edvardsson (1992). This is not surprising since the assumption that $[\mathrm{Ni} / \mathrm{Fe}]=0$ for all $[\mathrm{Fe} / \mathrm{H}]$ mainly results in a systematic shift of all abundances by a certain amount and will not affect the behaviour of the $[\mathrm{O} / \mathrm{Fe}]$ trend. Hence the Nissen et al. (2002) $[\mathrm{O} / \mathrm{Fe}]$ trend still level out at $[\mathrm{Fe} / \mathrm{H}]>0$.

\subsubsection{Evolution of the thick disk}

In Bensby et al. (2003) and Feltzing et al. (2003) we discuss different formation scenarios for the thick disk. Based on our elemental abundance trends in the thin and thick disks for the other $\alpha$-elements ( $\mathrm{Mg}, \mathrm{Si}, \mathrm{Ca}$, and $\mathrm{Ti}$ ) we concluded that it is very likely that the thin and thick disks formed at different epochs. This conclusion is further supported by the different mean stellar ages that we found from isochrones for the thin

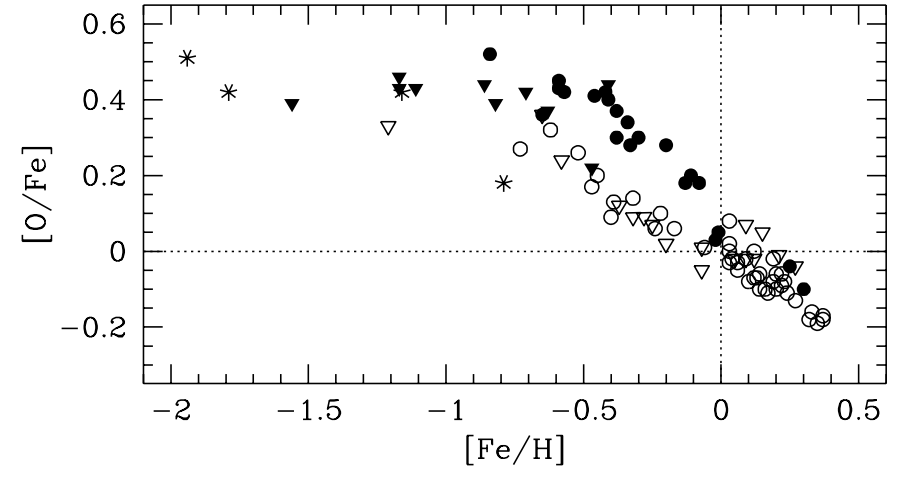

Fig. 11. Oxygen trends based on the $[\mathrm{O} I]_{6300}$ line for our stars and from the study by Nissen et al. (2002). The Nissen et al. (2002) thin and thick disk stars are marked by open and filled triangles, respectively, and their halo stars by asterisks. Our thin and thick disk stars are marked by open and filled circles, respectively.

and thick disk stars. The average ages are (including all stars in this study) $4.9 \pm 2.8$ and $10.8 \pm 4.3$ for our thin and thick disk stellar samples, respectively. However, our results could be accommodated both in a dissipational collapse scenario (either fast or slow) or in a merger/interacting scenario for the formation of the thick disk. However, evidence from extragalactic studies of edge-on spiral galaxies (e.g. Reshetnikov \& Combes 1997; Schwarzkopf \& Dettmar 2000; Dalcanton \& Bernstein 2002) appear to indicate that thick disks are more common in galaxies that are experiencing or have experienced mergers or interaction. Thus, at the moment the most plausible formation scenario for the thick disk is a merger event (e.g. Quinn et al. 1993) or an interaction with a companion galaxy (e.g. Kroupa 2002).

The thick disk shows the signatures of enrichment from SN Ia to the interstellar medium. This is clearly seen in plots where $[\mathrm{O} / \mathrm{Fe}]$ is plotted versus $[\mathrm{Fe} / \mathrm{H}]$ (Figs. 10a and 11). The "knee" in $[\mathrm{O} / \mathrm{Fe}]$ at $[\mathrm{Fe} / \mathrm{H}] \approx-0.4$ indicates when the SN Ia rate peaks and thereby also the peak in the enrichment of $\mathrm{Fe}$ from these events. That the "knee" is present at all also indicates that the star formation in the early thick disk has been vigorous. This can also be seen in Fig. 10b that shows how $[\mathrm{Fe} / \mathrm{O}]$ runs with $[\mathrm{O} / \mathrm{H}]$ as the uprising trend in $[\mathrm{Fe} / \mathrm{O}]$ at $[\mathrm{O} / \mathrm{H}] \sim 0$.

\subsubsection{Evolution of the thin disk}

The Galactic thin disk has not had such an intense star formation history as the thick disk. The shallow decline in the $[\mathrm{O} / \mathrm{Fe}]$ trend from an oxygen overabundance of $[\mathrm{O} / \mathrm{Fe}] \sim 0.2-0.3$ at $[\mathrm{Fe} / \mathrm{H}] \sim-0.8$ to an oxygen underabundance of $[\mathrm{O} / \mathrm{Fe}] \approx-0.2$ at $[\mathrm{Fe} / \mathrm{H}] \sim 0.4$ indicates a more continuous star formation with no fast initial enrichment from SN II. Instead the observed $[\mathrm{O} / \mathrm{Fe}]$ trend favours a chemical evolution in the thin disk where both SN Ia and SN II in a steady rate contribute to the enrichment of the interstellar medium and thereby producing a decline in $[\mathrm{O} / \mathrm{Fe}]$ with $[\mathrm{Fe} / \mathrm{H}]$ (see Fig. 10a).

How can we explain the trend observed in the thin disk? As noted in the preceding section the thin disk stars are, on average, younger than the thick disk stars. However, the thin disk stars appear to span the same metallicity range (below 


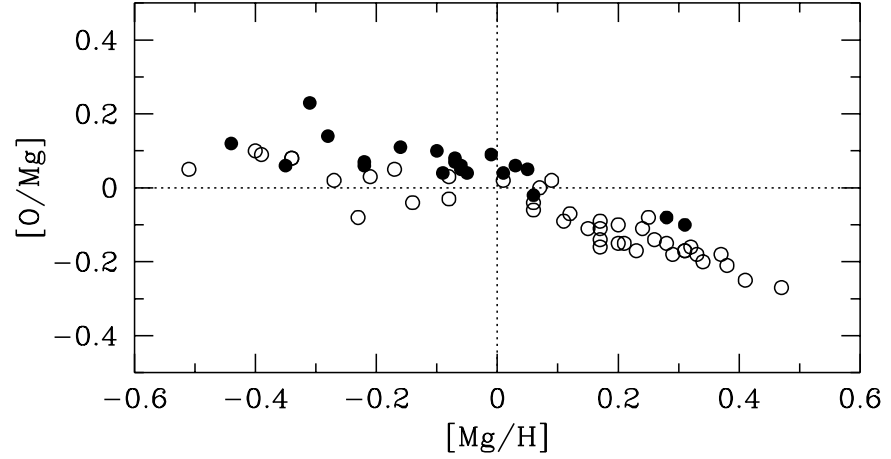

Fig. 12. $[\mathrm{O} / \mathrm{Mg}]$ vs. $[\mathrm{Mg} / \mathrm{H}]$. Only stars that have oxygen abundances from the $[\mathrm{OI}]_{6300}$ line are shown. $\mathrm{Mg}$ abundances are from Bensby et al. (2003). Thin and thick disk stars are marked by open and filled circles, respectively.

solar metallicity) as the thick disk stars do. A possible scenario for the star formation in the thin disk would be that once star formation in the thick disk was stopped (truncated?) there is a pause in the star formation. During this time there is in-falling fresh gas that accumulates in the Galactic plane, forming a new thin disk. Also the remaining gas from the thick disk will settle down onto the new disk. Once enough material is collected star formation is restarted in the new thin disk. The gas, though, has been diluted by the metal-poor in-falling gas. This means that the first stars to form in the thin disk will have lower metallicities than the last stars that formed in the thick disk. Although this scenario nicely explains the abundance trends in the thin disk it remains to work out the details and see if they fit other observational tests such as G dwarf and metallicity distributions.

Prior to this study the models of Galactic chemical evolution did not match the observed $[\mathrm{O} / \mathrm{Fe}]$ trend at $[\mathrm{Fe} / \mathrm{H}]>0$ since the models predict a downward trend (see e.g. Chiappini et al. 2003 for the most recent models) while the observed trend leveled out (see e.g. Nissen et al. 2002 and discussion in Sect. 7.1.1). Even though the models of Galactic chemical evolution rarely are evolved beyond $[\mathrm{Fe} / \mathrm{H}] \approx+0.2$ there is no indication of a leveling out.

\subsection{Synthesis of $\alpha$-elements and the choice of reference elements in chemical evolution studies}

The models of Galactic chemical evolution predict that $[\mathrm{Mg} / \mathrm{Fe}]$, at $[\mathrm{Fe} / \mathrm{H}]>0$, should show a downward trend similar to that seen for $[\mathrm{O} / \mathrm{Fe}]$. Our observations do not show this (Bensby et al. 2003). Instead of a downward trend [Mg/Fe] levels out at $[\mathrm{Fe} / \mathrm{H}] \approx 0$. In Fig. 12 we show $[\mathrm{O} / \mathrm{Mg}]$ as a function of $[\mathrm{Mg} / \mathrm{H}]$ which further emphasizes the different trends for oxygen and $\mathrm{Mg}$.

Prior to the new models by Meynet \& Maeder (2003) the models by Maeder (1992) predicted a strong dependency on the metallicity for the oxygen yields. This could then have explained the trend in Fig. 12. But, since the oxygen yields for massive stars now have been shown to be less sensitive to the metallicity when including rotation in the stellar models (Meynet \& Maeder 2003) this explanation is no longer viable. The stellar evolution models are also well-known to underestimate the $\mathrm{Mg}$ yields for massive stars (e.g. Chiappini et al.1999, 2003; Thomas et al. 1998) and thereby giving too low $[\mathrm{Mg} / \mathrm{Fe}]$ values at high $[\mathrm{Fe} / \mathrm{H}]$ in the models of Galactic chemical evolution. Currently, this could then be the explanation for the discrepancy between models of Galactic chemical evolution and observations for $\mathrm{Mg}$. However, this conclusion must remain tentative and more work on stellar yields is necessary for both $\mathrm{Mg}$ as well as oxygen in order to achieve a clear picture. In this our observational data can be used to further constrain the possible models.

In studies of chemical evolution of single or multiple stellar populations it is desirable to have a reference element that has only one, well-understood source. Stellar spectra are rich in easily measurable Fe lines. This means that for almost all stars Fe abundances are readily available and hence $\mathrm{Fe}$ is by far the most commonly used reference element in studies of galactic chemical evolution. However, $\mathrm{Fe}$ is produced in both SN II and SN Ia and therefore the trends are less easy to interpret than if we had a reference element that has only one source. Such reference elements could be provided by $\mathrm{Mg}$ or oxygen. However, in light of our own observations, the shortcomings of stellar yield models, and the fact that at least $\mathrm{Mg}$ might be produced, to some extent, in SN Ia we would be very cautious about promoting one specific reference element. It is probably best, for now, to consider more than one reference element.

\section{Summary}

We have presented oxygen abundances for 72 nearby $\mathrm{F}$ and $\mathrm{G}$ dwarf stars in the solar neighbourhood with $-0.9<[\mathrm{Fe} / \mathrm{H}]<+0.4$. The stellar samples were chosen in order to investigate two important issues related to the formation and chemical evolution of the stellar disks in our Galaxy:

$\star$ the oxygen trends in the thin and thick disk,

$\star$ the oxygen trend in the thin disk at super-solar metallicities.

The stars have been selected so that they either with a high likelihood belong to the thick or the thin disk. This selection was purely based on kinematics. The majority of the stars in the study presented here have previously been studied by Bensby et al. (2003) and Feltzing et al. (2003). In those two papers we derived stellar parameters, detailed statistics of the kinematic selection, and abundances for $\alpha$ - as well as iron peak-elements.

Our abundance analysis is based primarily on the forbidden oxygen line at $6300 \AA$. This line is blended with a doublet of $\mathrm{Ni}$ lines arising from two different $\mathrm{Ni}$ isotopes. As we have spectra of very high $S / N$ and $R$ and have modeled the line and its oxygen and $\mathrm{Ni}$ components in detail we have been able to disclose very well-defined trends for oxygen relative to iron and magnesium. We have also analyzed the forbidden line at $6363 \AA$ and the permitted triplet lines around $7774 \AA$ and found consistent trends from these lines.

Our main conclusions from this study are:

(i) At super-solar $[\mathrm{Fe} / \mathrm{H}]$ the $[\mathrm{O} / \mathrm{Fe}]$ trend for the thin disk continues linearly down-wards. This is different from the 
other $\alpha$-elements which show a leveling out of $[\alpha / \mathrm{Fe}]$ at $[\mathrm{Fe} / \mathrm{H}]=0$.

(ii) The thick disk is more overabundant in oxygen than the thin disk at sub-solar metallicities. The thick disk also shows the signatures of chemical enrichment from SN Ia.

(iii) Oxygen and magnesium do not evolve in lockstep at super-solar metallicities.

(iv) By comparing oxygen abundances from the permitted infrared O I triplet to those from the forbidden line at $6300 \AA$ we provide an empirical NLTE-correction relation for the abundances from the triplet lines that. This could be used e.g. for $\mathrm{F}$ and $\mathrm{G}$ dwarf star spectra with a $S / N$ that is such that only the triplet lines that can be analyzed well, e.g. due to the distances of the stars.

(v) Comparing our abundances from the $6300 \AA$ line and the triplet lines with and without NLTE corrections from Gratton et al. (1999) we find that their NLTE corrections, for the parameter space spanned by our stars, are somewhat overestimated.

Conclusion (i) is a new result and indicates that chemical evolution models of the Milky Way are now in concordance with the observed $[\mathrm{O} / \mathrm{Fe}]$ trends at $[\mathrm{Fe} / \mathrm{H}]>0$. The different trend for oxygen compared to the other $\alpha$-elements at $[\mathrm{Fe} / \mathrm{H}]>0$ (see Bensby et al. 2003) indicates that oxygen is only produced in SN II. The leveling out of the $[\alpha / \mathrm{Fe}]$ trend for other $\alpha$-elements could be expected on grounds that they also have small contributions from SN Ia. However, given the still large uncertainties in $\mathrm{SN}$ yield calculations this conclusion remains tentative.

Conclusion (ii) means that the Galactic thin and the thick disks indeed have different chemical histories. This is a confirmation and strengthening of previous findings by us and others (e.g. Prochaska et al. 2000). We also trace the signature of SN Ia (the "knee") in the $[\mathrm{O} / \mathrm{Fe}]$ trend in the thick disk that we have noticed previously for $\mathrm{Ca}, \mathrm{Mg}, \mathrm{Si}$, and $\mathrm{Ti}$ (Bensby et al. 2003; Feltzing et al. 2003).

Acknowledgements. We would like to thank Bengt Gustafsson, Kjell Eriksson, Bengt Edvardsson, and Martin Asplund for letting us use the Uppsala MARCS code. We further thank BG, KE and BE for the use of the EQWIDTH abundance program and the SPECTRUM line synthesis program. We would also like to thank Poul Erik Nissen, Bengt Gustafsson, and the anonymous referee for valuable comments on the submitted manuscript. TB also thanks Kungliga Fysiografiska Sällskapet i Lund for financial support to the first trip to La Silla in September 2000 and SF is grateful for computer grants from the same society. This research has made use of the SIMBAD database, operated at CDS, Strasbourg, France.

\section{References}

Abia, C., \& Rebolo, R. 1989, ApJ, 347, 186

Allende Prieto, C., Lambert, D. L., \& Asplund, M. 2001, ApJ, 556, L63

Andersson, H., \& Edvardsson, B. 1994, A\&A, 290, 590

Anstee, S. D., \& O'Mara, B. J. 1995, MNRAS, 276, 859

Asplund, M. 2003, CNO in the universe, ed. C. Charbonnel, D. Schaerer, \& G. Meynet, 10-14 September 2002 @ SaintLuc, Valais, Switzerland, ASP Conf. Ser., 304, in press [astro-ph/0302409]
Asplund, M., Gustafsson, B., Kiselman, D., \& Eriksson, K. 1997, A\&A, 318, 521

Balachandran, S. C., Carr, J. S., \& Carney, B. W. 2001, New Astron. Rev., 45, 529

Barbuy, B., \& Erdelyi-Mendes, M. 1989, A\&A, 214, 239

Barbuy, B., \& Nissen, P. E., Peterson, R. C., \& Spite, F. 2001, New Astron. Rev., 45, 509

Barklem, P. S., \& O'Mara, B. J. 1997, MNRAS, 290, 102

Barklem, P. S., \& O'Mara, B. J. 1998, MNRAS, 300, 863

Barklem, P. S., O’Mara, B. J., \& Ross, J. E. 1998, MNRAS, 296, 1057

Barklem, P. S., Piskunov, N., \& O'Mara, B. J. 2000, A\&AS, 142, 467

Bensby, T., Feltzing, S., \& Lundström, I. 2003, A\&A, 410, 527

Bessel, M. S., Sutherland, R. S., \& Ruan, K. 1991, ApJ, 383, L71

Biémont, E., \& Zeippen, C. J. 1992, 265, 850

Boesgaard, A. M., King, J. R., Deliyannis, C. P., \& Vogt, S. S. 1999, AJ, 117, 492

Carretta, E., Gratton, R. G., \& Sneden, C. 2000, A\&A, 356, 238

Castro, S., Rich, R. M., Grenon, M., Barbuy, B., \& McCarthy, J. K. 1997, AJ, 114, 376

Chen, Y. Q., Nissen, P. E., Zhao, G., Zhang, H. W., \& Benoni, T. 2000, A\&AS, 141, 491

Chen, Y. Q., Zhao, G., Nissen, P. E., Bai, G. S., \& Qiu, H. M. 2003, ApJ, 591, 925

Chiappini, C., Matteucci, F., Beers, T. C., \& Nomoto, K. 1999, ApJ, 515,226

Chiappini, C., Romano, D., \& Matteucci, F. 2003, MNRAS, 339, 63

Clegg, R. E. S., Lambert, D. L., \& Tomkin, J. 1981, ApJ, 250, 262

Dalcanton, J. J., \& Bernstein, R. A. 2002, AJ, 124, 1328

de Laverny, P., \& Gustafsson, B. 1998, A\&A, 332, 661

Edvardsson, B., Andersen, J., Gustafsson, B., et al. 1993, A\&A, 275, 101

Eriksson, K., \& Toft, S. C. 1979, A\&A, 71, 178

Feltzing, S., \& Gustafsson, B. 1998, A\&AS, 129, 237

Feltzing, S., Bensby, T., \& Lundström, I. 2003, A\&A, 397, L1

Gratton, R. G., \& Ortolani, S. 1986, A\&A, 169, 201

Gratton, R. G., Carretta, E., Eriksson, K., \& Gustafsson, B. 1999, A\&A, 350, 955

Gratton, R. G., Carretta, E., Matteucci, F., \& Sneden, C. 2000, A\&A, 358,671

Gratton, R. G., Carretta, E., Desidera, S., et al. 2003, A\&A, 406, 131

Grevesse, N., \& Sauval, A. J. 1998, Space Sci. Rev., 85, 161

Gustafsson, B., Bell, R. A., Eriksson, K., \& Nordlund, Å. 1975, A\&A, 42,407

Gustafsson, B., Karlsson, T., Olsson, E., Edvardsson, B., \& Ryde, N. 1999, A\&A, 342, 426

Hibbert, A., Biémont, E., Godefroid, M., \& Vaeck, N. 1991, J. Phys. B: At. Mol. Opt. Phys., 24, 3943

Israelian, G., García López, R. J., \& Rebolo, R. 1998, ApJ, 507, 805

Israelian, G., Rebolo, R., García López, R. J., et al. 2001, ApJ, 551, 833

Johansson, S., Litzén, U., Lundberg, H., \& Zhang, Z. 2003, ApJ, 584, L107

Kiselman, D. 1991, A\&A, 245, L9

Kiselman, D. 1993, A\&A, 275, 269

Kjærgaard, P., Gustafsson, B., Walker, G. A. H., \& Hultqvist, L. 1982, A\&A, 115, 145

Kroupa, P. 2002, MNRAS, 330, 707

Kupka, F., Piskunov, N., Ryabchikova, T. A., Stempels, H. C., \& Weiss, W. W. 1999, A\&AS, 138, 119

Kürster, M. 2001, The CES User Manual, Version 1.0, European Southern Observatory (ESO)

Lambert, D. L. 1978, MNRAS, 182, 249

Lambert, D. L., Sneden, C., \& Ries, L. M. 1974, ApJ, 188, 97 
Lide, D. R., 2002, CRC Handbook of Chemistry and Physics: a readyreference book of chemical and physical data, 83rd ed. (CRC Press)

Mäckle, R., Holweger, H., Griffin, R., \& Griffin, R. 1975, A\&A, 38, 239

Matteucci, F., \& Greggio, L. 1986, A\&A, 154, 279

Maeder, A. 1992, A\&A, 264, 105

Meléndez, J., \& Barbuy, B. 2002, ApJ, 575, 474

Meléndez, J., Barbuy, B., \& Spite, F. 2001, ApJ, 556, 858

Meynet, G., \& Maeder, A. 2003, A\&A, 390, 561

Mishenina, T. V., Korotin, S. A., Klochkova, V. G., \& Panchuk, V. E. 2000, A\&A, 353, 978

Mitchell, W. E. Jr., \& Mohler, O. C. 1965, ApJ, 141, 1126

Moore, C. E., Minnaert, M. G. J., \& Houtgast, J. 1966, The Solar Spectrum $2935 \AA$ to $8770 \AA$ A, National Bureau of Standards Monograph 61

Nave, G., Johansson, S., Learner, R. C. M., Thorne, A. P., \& Brault, J. W. 1994, ApJS, 94, 221

Naqvi, A. M. 1951, Thesis Harvard

Nissen, P. E., \& Schuster, W. J. 1997, A\&A, 326, 751

Nissen, P. E., \& Edvardsson, B. 1992, A\&A, 261, 255

Nissen, P. E., Primas, F., Asplund, M., \& Lambert, D. L. 2002, A\&A, 390, 235

Omholt, A. 1960, Planetary and Space Science, 2, 246

Piskunov, N. E., Kupka, F., Ryabchikova, T. A., Weiss, W. W., \& Jeffery, C. S. 1995, A\&AS, 112, 525
Pompéia, L., Barbuy, B., \& Grenon, M. 2002, ApJ, 566, 845

Prochaska, J. X., Naumov, S. O., Carney, B. W., McWilliam, A., \& Wolfe, A. M. 2000, ApJ, 120, 2513

Quinn, P. J., Hernquist, L., \& Fullagar, D. P. 1993, ApJ, 403, 74

Reddy, B. E., Tomkin, J., Lambert, D. L., \& Allende Prieto, C. 2003, MNRAS, 340, 304

Reshetnikov, V., \& Combes, F. 1997, A\&A, 324, 80

Ryabchikova, T. A., Piskunov, N. E., Stempels, H. C., Kupka, F., \& Weiss, W. W. 1999, Proc. of the 6th Intern. Colloq. on Atomic Spectra and Oscillator Strengths, Victoria BC, Canada, 1998, Physica Scripta, T83, 162 (VALD-2)

Schwarzkopf, U., \& Dettmar, R.-J. 2000, A\&A, 361, 451

Shi, J. R., Zhao, G., \& Chen, Y. Q. 2002, A\&A, 381, 982

Sneden, C., Lambert, D. L., \& Whitaker, R. W. 1979, ApJ, 234, 964

Stoffregen, W., \& Derblom, H. 1960, Nature, 185, 28

Tautvaišienė, G., Edvardsson, B., Tuominen, I., \& Ilyin, I. 2001, A\&A, 380, 579

Thomas, D., Greggio, L., \& Bender, R. 1998, MNRAS, 296, 119

Tinsley, B. M. 1979, ApJ, 229, 1046

Wheeler, J. C., Sneden, C., \& Truran, Jr. J. W. 1989, ARA\&A, 27, 279

Wiese, W. L., Smith, M. W., \& Glennon, B. M. 1966, Atomic Transition Probabilities, vol. 1 Hydrogen Through Neon, National Bureau of Standards 4 (NSRDS-NBS 4)

Yamanouchi, T., \& Horie, H. 1952, J. Phys. Soc. Jpn, 7, 52 